\title{
A Plastic-Damage Model for Lightweight Concrete and Normal Weight Concrete
}

\author{
C. G. Koh, ${ }^{1)}$ M. Q. Teng, ${ }^{2)}$ and T. H. Wee ${ }^{2)}$
}

(Received June 12, 2008, Revised October 27, 2008, Accepted November 30, 2008)

\begin{abstract}
A new plastic-damage constitutive model applicable to lightweight concrete (LWC) and normal weight concrete (NWC) is proposed in this paper based on both continuum damage mechanics and plasticity theories. Two damage variables are used to represent tensile and compressive damage independently. The effective stress is computed in the Drucker-Prager multi-surface plasticity framework. The stress is then computed by multiplication of the damaged part and the effective part. The proposed model is coded as a user material subroutine and incorporated in a finite element analysis software. The constitutive integration algorithm is implemented by adopting the operator split involving elastic predictor, plastic corrector and damage corrector. The numerical study shows that the algorithm is efficient and robust in the finite element analysis. Experimental investigation is conducted to verify the proposed model involving both static and dynamic tests. The very good agreement between the numerical results and experimental results demonstrates the capability of the proposed model to capture the behaviors of LWC and NWC structures for static and impact loading.
\end{abstract}

Keywords : lightweight concrete, continuum damage mechanics, plasticity, finite element analysis, operator split

\section{Introduction}

Lightweight concrete (LWC) provides high strength-to-weight ratio and is ideal for long span structures, super highrise buildings and offshore floating structures. For example, in Norway, LWC has been successfully used in offshore structures for oil drilling platforms, storage tanks and vessels LWC also offers other advantages such as good thermal insulation, sound insulation and fire resistance. $^{2}$ For design and analysis of such structures, numerical methods are often used, among which the finite element method (FEM) is the most commonly adopted method. The validity of the numerical results largely depends on accurate material constitutive models which are used at two stages of the FEM: evaluation of stresses and evaluation of tangent stress-strain matrices. ${ }^{3}$ Thus, research on constitutive relation is essential to the analysis and design of LWC as well as normal weight concrete (NWC) structures.

Concrete is primarily a composite material composed of aggregate material embedded in a hard matrix of material (cement or binder) that fills the space between the aggregates and glues them together. Therefore, many researchers ${ }^{4-6}$ used micromechanics to model microcrack growth in the original brittle bodies based on the properties of the constituents alone. However, $\mathrm{Ju}^{7}$ pointed out

\footnotetext{
${ }^{1)}$ National University of Singapore, Dept. of Civil Engineering, Singapore 117576. E-mail: cgkoh@nus.edu.sg

${ }^{2)}$ National University of Singapore, Dept. of Civil Engineering, Singapore 117576.

Copyright (c) 2008, Korea Concrete Institute. All rights reserved, including the making of copies without the written permission of the copyright proprietors.
}

that a purely micromechanical theory may never replace a properly formulated phenomenological modeling theory due to its intrinsic complexity. Consequently, the phenomenological model on macromechanical scale provides an attractive means for numerical modeling of concrete structures without the need of detailed microstructure modeling of material. Although there are numerous constitutive models proposed for NWC on macromechanical scale, there is insufficient research on constitutive modeling of LWC particularly since LWC is a relatively new material.

There have been many different ways to model the constitutive behaviors of concrete, e.g. elasticity theory, classical plasticity theory, endochronic theory, fracture mechanics, continuum damage mechanics (CDM) model and mixed (hybrid) models. Among these, the more popular models are based on plasticity, fracture mechanics and CDM. Historically, plasticity was originally developed for metals and is now also applied to concrete because of its sound theoretical basis and favorable computational efficiency. 8,9 The plasticity model clearly has advantages over elastic approaches in representing hardening and softening characteristics. Nevertheless, it does not explicitly incorporate damage process due to microcracks such as stiffness degradation and unilateral effects. A good coverage of concrete plasticity models can be found in the book written by Chen. ${ }^{10}$

Fracture mechanics model was also originally developed for metals ${ }^{11}$ and later extended to concrete. ${ }^{12}$ Much effort is still being devoted to refine fracture mechanics models for concrete. Extensive reviews of concrete fracture mechanics can be found in the literature. $^{13,14}$ Despite some successful applications of fracture mechanics on concrete, whether or not the $J$ integrals and stress intensity factor $K$ are material parameters remains a controversial issue. Furthermore, for practical application, it is difficult to define 
the geometry and location of the microcracks precisely before they are formed.

When concrete damage is involved, an alternative approach of modeling is to use CDM based on the thermodynamics of irreversible process. Early CDM models for concrete were developed by Mazars and Pijaudier-Cabot ${ }^{15}$ and Cervera et al. ${ }^{16}$ Though these models do handle phenomena such as stiffness degradation, strain softening and unilateral effects, they are confined only in the elastic ranges and cannot correctly describe the nonlinear behavior of concrete. To account for the nonlinear behavior, more concrete models have been developed in the framework of nonlinear CDM. These models fall under three categories according to how damage criteria are defined: equivalent strain approaches ${ }^{17}$; stressbased approaches; ${ }^{18,19}$ damage energy release rate (DERR) based approaches. ${ }^{7,17,20}$ Equivalent strain-based criteria are only appropriate for the elastic conditions whereas stress-based approaches are unable to predict the plastic damage growth accurately. ${ }^{7}$ Most of concrete damage models based on DERRs only consider elastic Helmoholtz free energy which is only the elastic part of the potential energy. ${ }^{19,21}$ Though damage elastoplastic models incorporating plastic strain and plastic Helmoholtz free energy are more reasonable, the plastic Helmoholtz free energy can be formulated explicitly only for simple situations such as Von Mises model with isotropic linear hardening rule and its numerical algorithm is rather complicated and possibly unstable. ${ }^{7}$

In short, CDM is versatile in handling many of the observed phenomena including stiffness degradation, tensile softening, and unilateral effects due to microcracks and microvoids. But if the inelastic (plastic) strain is not considered, the model cannot predict some behaviors such as diltancy which is very important for multiaxial loadings. ${ }^{10,22}$ A complementary approach, namely the plasticity theory, is needed for modeling the inelastic strains. Furthermore, it is well-recognized that the macroscale response of concrete originates mainly from (a) the initiation, growth and coalescence of microcracks, and (b) the pressure-dependent (frictional) tangential movement of the microcrack surfaces. Since the kinetics of the microcracks can be accounted for by the theory of CDM and frictional tangential motion can be modeled by pressure-sensitive plasticity theory, it is justifiable and promising to combine these two different ways of modeling concrete behavior and capturing the microscale mechanisms as realistically as possible. ${ }^{23}$

In this paper, a new plastic-damage constitutive model is developed with the intention of applying it to not just NWC but also LWC under investigation. The numerical algorithm to implement this model in the framework of FEM is explained. In addition, experimental investigation is carried out to obtain the necessary material parameters and to verify the applicability of the proposed model under different loading conditions including impact tests.

\section{Plastic-damage model}

The objective herein is to study the response at structural level and it is reasonable to treat cracks in a smeared way. To this end, the CDM model is a phenomenological model accounting for the damage effect of cracks in the constitutive equation rather than dealing with individual cracks. The model is assumed to be developed in the framework of infinitesimal deformation theory, which is adequate for modeling concrete materials. ${ }^{22}$ As explained ear- lier, in view of the advantages of plasticity model and CDM, it is desirable to combine these two approaches for constitutive modeling of concrete. In the present model, the effective stress in CDM is used to simulate stiffness degradation as in Ju's model. ${ }^{7}$ This decouples the damage in stiffness degradation from the plasticity deformations. Therefore, it is very convenient to implement the operator split and to divide the computational algorithm into three steps: elastic predictor, plastic corrector and damage corrector. In the FE model, the degradation of stiffness is handled at each integration point.

The plastic deformations are computed in the effective stress space, independent of the damage corrector step for evaluation of the damage in stiffness degradation. In the plastic part, the model comprises two Drucker-Prager-type yielding surfaces: one for compression and the other for tension. The associated plastic flow and isotropic hardening rules are adopted. In the CDM part, two internal parameters are defined for tensile damage and compressive damage. At the final stage, one scalar damage parameter is proposed by combination of tensile and compressive damage parameters to describe both tensile and compressive damages.

\subsection{Main significance of the model}

It is important to note that the proposed constitutive model contains only a few parameters that can be readily determined by standard laboratory tests. This model incorporates two DruckerPrager-type yielding surfaces, which can account for the important phenomenon of diltancy not considered in many other mixed models employing relatively simple plasticity criteria, e.g. Von Mises criterion. Yet this proposed model is relatively easy for implementation in the finite element analysis and, as will be shown later, accurate when compared to the experimental results obtained for both static and impact loading.

\subsection{Theoretical framework}

In the small strain plasticity theory, the strain $\varepsilon$ is decomposed into elastic part $\varepsilon^{e}$ and plastic part $\varepsilon^{p}$ :

$$
\varepsilon=\varepsilon^{e}+\varepsilon^{p}
$$

In linear elasticity, the constitutive equation is given by

$$
\sigma=\mathrm{C}:\left(\varepsilon-\varepsilon^{p}\right)
$$

where, $\sigma$ is the stress tensor and $C$ is the elastic stiffness tensor. In accordance with CDM, the stress tensor $\sigma$ can be mapped into the effective stress tensor $\bar{\sigma}$ by the relation:

$$
\bar{\sigma}=T: \sigma
$$

where $T$ is a rank-four mapping tensor. Note that the effective stress is defined in undamaged elastic stiffness. Thus

$$
\bar{\sigma}=C_{0}:\left(\varepsilon-\varepsilon^{p}\right)
$$

where, $C_{0}$ is the initial elastic stiffness.

In this study, a scalar damage variable $D$ is used to describe the isotropic damage such that $T=\frac{1}{(1-D)} I$, where $I$ is the rank-four

identity tensor. From Eq. (3), we obtain

$$
\sigma=(1-D) \bar{\sigma}
$$

Comparing Eq. (2) and Eq. (4) gives: 


$$
C=(1-D) C_{0}
$$

Experimental observations indicate that tensile damage and compressive damage of concrete give very different responses. ${ }^{22}$ To account for this difference, two damage variables are needed: $d_{t}$ and $d_{c}$ representing the tensile damage and compressive damage, respectively. The uniaxial degradation variables are increasing functions of the equivalent plastic strains. They can take values ranging from zero, for the undamaged material, to one, for the fully damaged material. Theoretically, they can be obtained by uniaxial tests as function of the equivalent plastic strains. In practice, however, such experiments are difficult, if not impossible, to conduct and thus the explicit formulations for $d_{t}$ and $d_{c}$ are not readily available. To handle this problem, many researchers assume that the degradation takes the following exponential form $^{22,24}$ :

$$
\left\{\begin{array}{l}
d_{c}=1-\exp \left(-a_{c} \varepsilon^{p}\right) \\
d_{t}=1-\exp \left(-a_{t} \varepsilon^{p}\right)
\end{array}\right.
$$

where $a_{t}$ and $a_{c}$ are model parameters for uniaxial tension and compression, respectively, and can be calibrated from the uniaxial tensile and compressive tests by imposing the conditions:

$$
\left\{\begin{array}{l}
\left.d_{c}\right|_{\varepsilon_{\varepsilon}^{p}=\varepsilon_{l, \text { max }}^{p}}=1 \\
\left.d_{t}\right|_{\varepsilon_{\varepsilon_{l}^{p}}=\varepsilon_{l, \text { max }}^{p}}=1
\end{array}\right.
$$

The scalar damage variable $D$ in Eq. (5) is given as ${ }^{22,24}$ :

$$
D=1-\left(1-d_{t}\right)\left(1-d_{c}\right)
$$

From Eqs. (1), (7) and (9), the damage evolution $(D-\varepsilon)$ relation can be obtained. Fig. 1 shows typical damage evolution curves for LWC and NWC under uniaxial compression based on experimental results. It is seen that damage in NWC is found to increase gradually. In contrast, the damage in LWC occurs and increases rapidly in a narrow strain range due to its relative higher brittleness.

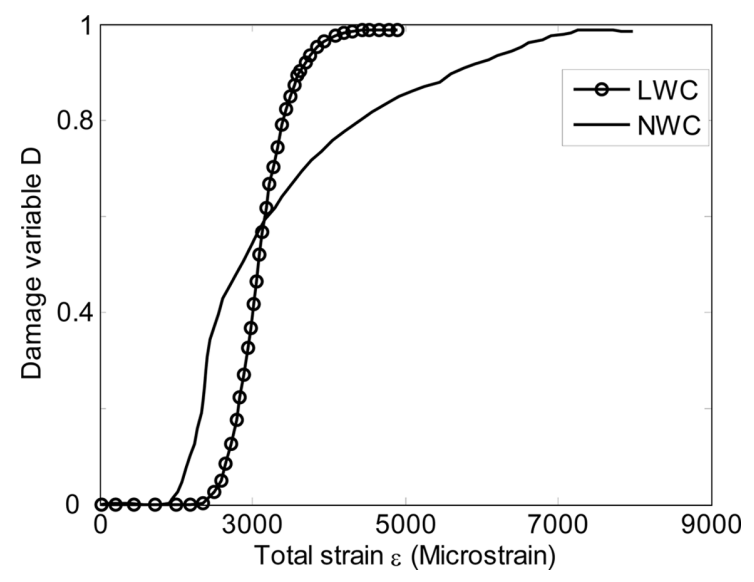

Fig. 1 Typical damage evolution curves for LWC and NWC.
The general Drucker-Prager yielding surface is described by

$$
F=\alpha I_{1}+\sqrt{J_{2}}-\tau_{0}=0
$$

where, $\alpha$ and $\tau_{0}$ are model parameters, $I_{1}=\sigma_{11}+\sigma_{22}+\sigma_{33}$ is the first invariant of the stress tensor $\sigma_{i j}(i, j=1,2,3)$ and $J_{2}$ is the second deviatoric invariant of the stress tensor $\sigma_{i j}(i, j=1,2,3)$, which is defined as:

$$
\begin{aligned}
& J_{2}=\frac{1}{6}\left[\left(\sigma_{11}-\sigma_{22}\right)^{2}+\left(\sigma_{11}-\sigma_{33}\right)^{2}+\left(\sigma_{22}-\sigma_{33}\right)^{2}\right] \\
& +\sigma_{12}^{2}+\sigma_{23}^{2}+\sigma_{13}^{2}
\end{aligned}
$$

The values of $I_{1}$ and $J_{2}$ at the peak stress for uniaxial compression and tension (denoted by subscripts $c$ and $t$ respectively) are

$$
\left\{\begin{array}{l}
I_{1 c}=-f_{c}, J_{2 c}=\frac{f_{c}^{2}}{3} \\
I_{1 t}=f_{t}, J_{2 t}=\frac{f_{t}^{2}}{3}
\end{array}\right.
$$

where, $f_{c}$ and $f_{t}$ are uniaxial compressive strength and tensile strength, respectively. Substituting Eq. (12) into Eq. (10) gives:

$$
\left\{\begin{array}{l}
-\alpha_{1} f_{c}+\frac{f_{c}}{\sqrt{3}}-\tau_{01}=0 \\
\alpha_{1} f_{t}+\frac{f_{t}}{\sqrt{3}}-\tau_{01}=0
\end{array}\right.
$$

where, $\alpha_{1}$ and $\tau_{01}$ are the model parameters for the first yielding surface $F_{1}$ :

$$
\alpha_{1}=\frac{1}{\sqrt{3}} \frac{f_{c}-f_{t}}{f_{c}+f_{t}} ; \quad \tau_{01}=\frac{2}{\sqrt{3}} \frac{f_{c} f_{t}}{f_{c}+f_{t}}
$$

The failure envelope $F_{1}$ in the biaxial tension and tension-compression quadrants (Fig. 2) can be obtained:

$$
F_{1}=\alpha_{1} I_{1}+\sqrt{J_{2}}-\tau_{01}=0
$$

In the same way, by matching the uniaxial and equibiaxial com-

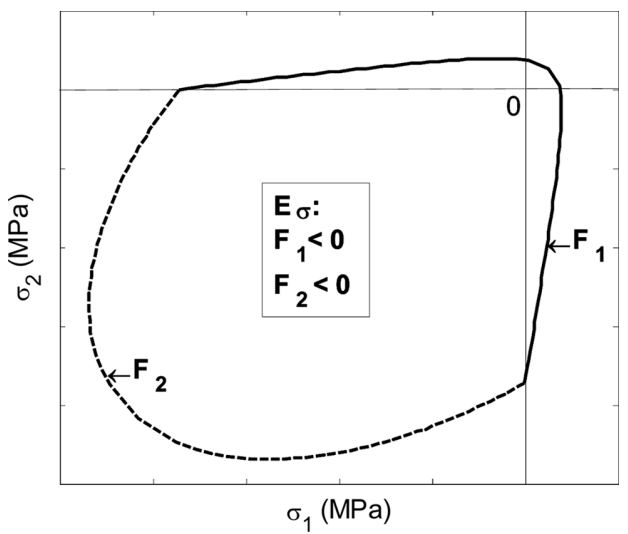

Fig. 2 Drucker-Prager yield surface in plane stress space. 
pressive strength to $I_{1}$ and $J_{2}$ and solving for $\alpha_{2}$ and $\tau_{02}$, we can obtain the yielding envelope in the biaxial compression quadrant:

$$
\begin{aligned}
& F_{2}=\alpha_{2} I_{1}+\sqrt{J_{2}}-\tau_{02}=0 \\
& \alpha_{2}=\frac{1}{\sqrt{3}} \frac{f_{b}-f_{c}}{2 f_{b}+f_{c}} ; \tau_{02}=\frac{1}{\sqrt{3}} \frac{f_{b} f_{c}}{f_{b}-f_{c}}
\end{aligned}
$$

where, $f_{b}$ is the equibiaxial compressive strength. Under plane stress conditions $\left(\sigma_{3}=0\right)$, Eq. (10) can be written as:

$$
\left(1-3 \alpha^{2}\right)\left(\sigma_{1}^{2}+\sigma_{1}^{2}\right)-\left(1+6 \alpha^{2}\right) \sigma_{1} \sigma_{2}+6 \tau_{0} \alpha\left(\sigma_{1}+\sigma_{2}\right)-3 \tau_{0}^{2}=0
$$

or in the form:

$$
A \sigma_{1}^{2}+B \sigma_{1} \sigma_{2}+C \sigma_{2}^{2}+D \sigma_{1}+E \sigma_{2}+F=0
$$

where, $A=1-3 \alpha^{2}, B=-\left(1+6 \alpha^{2}\right), C=1-3 \alpha^{2}, D=6 \tau_{0} \alpha, E=$ $6 \tau_{0} \alpha$, and $F=-3 \tau_{0}^{2}$. Depending on the coefficients in Eq. (19), the equation can be elliptic, parabolic or hyperbolic. For yielding surface $F_{1}$, we have:

$$
B^{2}-4 A C=12\left(\frac{f_{c}-f_{t}}{f_{c}+f}\right)^{2}-3
$$

The critical value for which Eq. (18) describes a parabola is $B^{2}-$ $4 A C=0$, i.e. $f_{t} / f_{c}=1 / 3$. For concrete, the value of ratio $f_{t} / f_{c}$ is usually less than $1 / 10$. Thus, the intersection of the DruckerPrager cone of yielding surface $F_{1}$ with the biaxial stress plane is a hyperbola (Fig. 2).

The material parameters required for the concrete model are Young's modulus, Possion's ratio, compressive strength, tensile strength and equibiaxial strength. Except of equibiaxial strength, all the other material parameters can be determined by commonly conducted tests. Kupfer et al. ${ }^{25}$ performed the classical study for the biaxial loading for concrete and showed that the ratio of equibiaxial strength to compression strength $\left(f_{b} / f_{c}\right)$ ranges from 1.10 to 1.20. Lubliner et al. ${ }^{24}$ also reported that this ratio is about 1.10 to 1.16. Lee and Fenves ${ }^{26}$ and $\mathrm{Wu}^{27}$ found that the result is not sensitive to this ratio within the range. In this study, $f_{b} / f_{c}=1.12$ is adopted. Consequently, $\alpha_{2}=0.021383$ and $B^{2}-4 \mathrm{AC}=-2.98<0$ which means that the intersection of the Drucker-Prager cone of yielding surface $F_{2}$ with the biaxial stress plane is an ellipse with a shifted center (see Fig. 2).

Fig. 2 shows that the proposed model is non-smooth multi-surface model and gradient is not uniquely defined at the intersection points of the two yielding surfaces. This problem can be handled by using Koiter's rule ${ }^{28}$ as follows

$$
\varepsilon^{p}=\sum_{\alpha}^{2} \gamma^{\alpha} \partial_{\bar{\sigma}} F_{\alpha}(\bar{\sigma}, \kappa)
$$

where, $\partial_{\bar{\sigma}} F_{\alpha}=\frac{\partial F_{\alpha}}{\partial \bar{\sigma}}$ and $\gamma^{\alpha}$ is the plastic consistent parameter.

Uniaxial compressive and tensile stress-strain curves based on experimental observations are adopted as shown in Figs. 3 and 4, respectively. Under uniaxial compression, the stress-strain relation is linear until the initial yield $\left(f_{c 0}\right)$ followed by the plastic regime, which is characterized by plastic hardening. Beyond the ultimate stress $f_{c}$ is the strain softening part of the stress-strain curve. Under uniaxial tension the stress-strain relation is linear until the failure stress $\left(f_{t 0}\right)$ followed by strain softening. Isotropic hardening is assumed such that the uniaxial stress-strain curves can be converted into stress versus plastic-strain curves of the form:

$$
\begin{aligned}
& f_{t}=f_{t}\left(\tilde{\varepsilon}_{t}^{p}\right) \\
& f_{c}=f_{c}\left(\tilde{\varepsilon}_{c}^{p}\right)
\end{aligned}
$$

where the equivalent plastic strains are:

$$
\begin{aligned}
& \tilde{\varepsilon}_{t}^{p}=\int_{0}^{t} \tilde{\dot{\varepsilon}}_{t}^{p} d t \\
& \tilde{\varepsilon}_{c}^{p}=\int_{0}^{t} \dot{\varepsilon}_{c}^{p} d t
\end{aligned}
$$

Lubliner et al. $^{24}$ assumed exponential forms for hardening and softening. In this paper, the stress versus plastic-strain curves is obtained by the following procedure.

From Eq. (1), the plastic strain can be obtained:

$$
\varepsilon_{i}^{p}=\varepsilon_{i}-\varepsilon_{i}^{e}(i=t, c)
$$

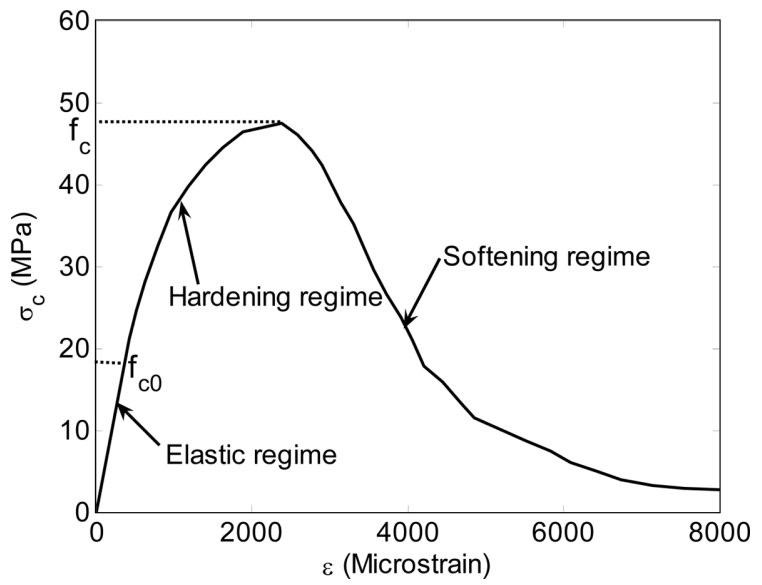

Fig. 3 Typical stress-strain relationship for concrete under compression.

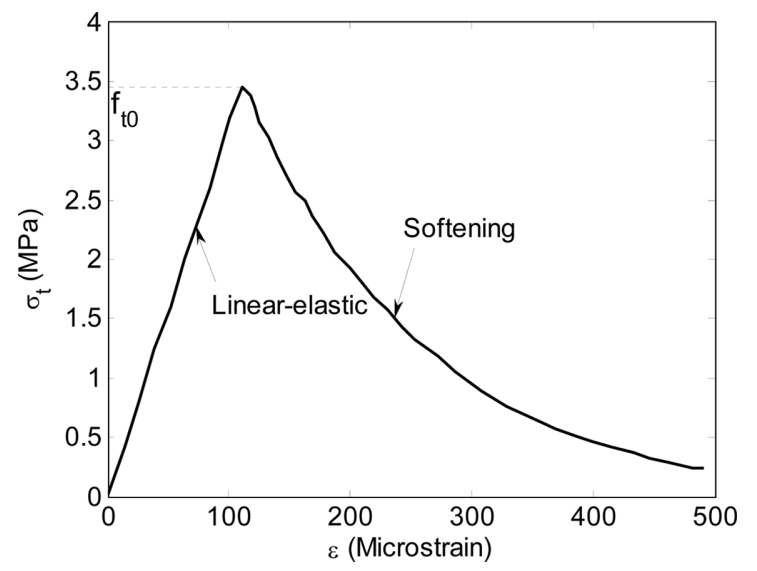

Fig. 4 Typical stress-strain relationship for concrete under tension. 
Since elastic strain $\varepsilon_{i}^{e}=\frac{f_{i 0}}{E}$, Eq. (24) can be rewritten as:

$$
\varepsilon_{i}^{p}=\varepsilon_{i}-\frac{f_{i 0}}{E}(i=t, c)
$$

Therefore, stress versus plastic-strain can be derived from the uniaxial stress-strain curves obtained by uniaxial compressive and tensile tests.

The hardening rules for the uniaxial conditions must be extended to the general multiaxial conditions. Making use of the findings by Lee and Fenves ${ }^{22}$ and $\mathrm{ABAQUS}^{29}$, the equivalent plastic strain for the general multiaxial conditions is expressed as:

$$
\begin{aligned}
& \dot{\tilde{\varepsilon}}_{t}^{p}=r(\hat{\bar{\sigma}}) \hat{\dot{\varepsilon}}_{\max }^{p} \\
& \dot{\tilde{\varepsilon}}_{t}^{p}=-(1-r(\hat{\bar{\sigma}})) \hat{\dot{\varepsilon}}_{\min }^{p}
\end{aligned}
$$

where, $\hat{\dot{\varepsilon}}_{\text {max }}^{p}$ and $\hat{\dot{\varepsilon}}_{\text {min }}^{p}$ are maximum and minimum eigenvalues of plastic strain rate tensor $\dot{\varepsilon}^{p}$ and

$$
r(\hat{\bar{\sigma}}) \stackrel{\operatorname{def}_{=}^{3}\left\langle\hat{\bar{\sigma}}_{i}\right\rangle}{\sum_{i=1}^{3}\left|\hat{\bar{\sigma}}_{i}\right|} ; 0 \leq r(\hat{\bar{\sigma}}) \leq 1
$$

is a weight factor between zero and one. The weight factor is equal to one if all principal stress components $\hat{\bar{\sigma}}_{i}(i=1,2,3)$ are positive and equal to zero if all are negative. The sign $\langle$.$\rangle denotes the$ Macauley bracket defined by $\langle x\rangle=\frac{1}{2}(|x|+x)$. It is obvious that, under uniaxial conditions, Eq. (26) can be reduced to uniaxial definitions since $\hat{\dot{\varepsilon}}_{\text {max }}^{p}=\hat{\dot{\varepsilon}}_{11}^{p}$ in tension, and $\hat{\dot{\varepsilon}}_{\text {max }}^{p}=\hat{\dot{\varepsilon}}_{11}^{p}$ in com-pression.

For ease of reference, the eigenvalues of the plastic strain rate tensor $\dot{\varepsilon}^{p}$ are ordered such that $\hat{\dot{\varepsilon}}_{\text {max }}^{p}=\hat{\dot{\varepsilon}}_{1}^{p} \geq \hat{\dot{\varepsilon}}_{2}^{p} \geq \hat{\dot{\varepsilon}}_{3}^{p}=\hat{\dot{\varepsilon}}_{\text {min }}^{p}$. Therefore, the hardening rules for the general multiaxial conditions are expressed in the following form:

$$
\dot{\tilde{\varepsilon}}^{p}=\left\{\begin{array}{l}
\hat{\dot{\varepsilon}}_{t}^{p} \\
\hat{\dot{\varepsilon}}_{c}^{p}
\end{array}\right\}=h\left(\hat{\bar{\sigma}}, \tilde{\varepsilon}^{p}\right) \cdot \hat{\varepsilon}^{p}
$$

where

$$
h\left(\hat{\bar{\sigma}}, \tilde{\varepsilon}^{p}\right)=\left[\begin{array}{ccc}
r(\hat{\bar{\sigma}}) & 0 & 0 \\
0 & 0-(1-r(\hat{\bar{\sigma}}))
\end{array}\right]
$$

and

$$
\hat{\dot{\varepsilon}}^{p}=\left\{\begin{array}{l}
\hat{\dot{\varepsilon}}_{1} \\
\hat{\dot{\varepsilon}}_{2} \\
\hat{\dot{\varepsilon}}_{3}
\end{array}\right\}
$$

In principal stress space, the flow rule is written as:

$$
\hat{\dot{\varepsilon}}^{p}=\sum_{a=1}^{2} \dot{\gamma}_{\alpha} \partial_{\hat{\bar{\sigma}}} \hat{F}_{\alpha}
$$

Substituting Eq. (31) into Eq. (28) gives:

$$
\dot{\tilde{\varepsilon}}^{p}=\left\{\begin{array}{c}
\hat{\varepsilon}_{t}^{p} \\
\hat{\varepsilon}_{t}^{p}
\end{array}\right\}=h\left(\hat{\bar{\sigma}}, \overline{\bar{\varepsilon}}^{p}\right) \cdot\left\{\begin{array}{c}
\sum_{\alpha=1}^{2} \dot{\gamma}_{\alpha} \partial_{\bar{\sigma}_{1}} \hat{F}_{\alpha} \\
2 \\
\sum_{\alpha=1}^{2} \dot{\gamma}_{\alpha} \partial_{\bar{\sigma}_{2}} \hat{F}_{\alpha} \\
2 \\
\sum_{\alpha=1}^{2} \dot{\gamma}_{\alpha} \partial_{\bar{\sigma}_{3}} \hat{F}_{\alpha}
\end{array}\right\}=\sum_{\alpha=1}^{2} \dot{\gamma}_{\alpha} \cdot H_{\alpha}\left(\hat{\bar{\sigma}}, \tilde{\varepsilon}^{p}\right)
$$

where

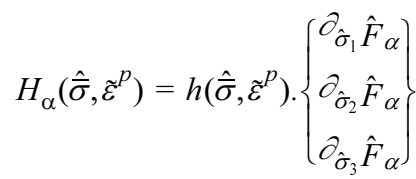

is the hardening function for this model. For convenience, hardening parameters $\kappa$ is defined as:

$$
\kappa=\left\{\begin{array}{l}
\kappa_{t} \\
\kappa_{c}
\end{array}\right\}=\varepsilon^{p}
$$

where $\kappa_{t}$ and $\kappa_{c}$ are hardening parameters for tension and compression, respectively.

\section{Numerical algorithm}

The numerical solution procedure based on the FEM is well covered by many books. ${ }^{30-32}$ For nonlinear problems, the equilibrium equations are satisfied by iterative methods at the global (structure) level. At the local (element) level, the nonlinear constitutive equations are solved by, in this study, an operator split procedure involving elastic predictor, plastic corrector and damage corrector. The key steps of the solution procedure are outlined here.

The weak form of the linear momentum equation at time $t_{n+1}$ in a discrete system can be written as the nonlinear equation for displacement $u_{n+1}$ at time $t_{n+1}$ :

$$
F^{\mathrm{int}}\left(u_{n+1}\right)-F^{\mathrm{ext}}\left(u_{n+1}\right)=0
$$

where, $F^{\text {int }}$ is the internal forces and $F^{\text {ext }}$ is the external forces, which are evaluated by

$$
F^{\mathrm{int}}=\int_{\Omega} B^{T} \sigma_{n+1} d \Omega
$$

where, $B$ is the strain-displacement matrix such that

$$
\varepsilon_{n+1}=B u_{n+1}
$$

The stress $\sigma_{n+1}$ is given by

$$
\sigma_{n+1}=\sigma_{n}+f_{\sigma}\left(B u_{n+1}, \sigma_{n+1}\right)
$$

Since $f_{\sigma}$ is a highly nonlinear function, an iterative scheme such as Newton-Raphson method should be employed to solve Eq. (35) with the residual defined as follows

$$
\Phi=F^{\mathrm{int}}\left(u_{n+1}\right)-F^{\mathrm{ex} t}\left(u_{n+1}\right)
$$


Linearization of Eq. (39) with $u_{n+1}^{0}=u_{n}$, at the $k$-th iteration gives

$$
\begin{aligned}
& \Phi^{(k)}+\left(\frac{d \Phi}{d u_{n+1}}\right)^{k} \delta u=0 \\
& u_{n+1}^{(k)}=u_{n+1}^{(k-1)}+\delta u
\end{aligned}
$$

where the derivative of the residual with respect to the displacement $\frac{d \Phi}{d u_{n}+1}$ is the tangent stiffness matrix $K$ for the NewtonRaphson method, and has the following form:

$$
\frac{d \Phi}{d u_{n+1}}=\int_{\Omega} B^{T} \frac{d \sigma_{n+1}}{d \varepsilon_{n+1}} B d \Omega
$$

where $\frac{d \sigma_{n+1}}{d \varepsilon_{n+1}}$ is the consistent tangent modulus (or Jacobian matrix) which is often used instead of the continuum elasto-plastic tangent modulus to preserve quadratic convergence rate in the fully implicit backward Euler scheme during integration of constitutive equations $^{20,32,33}$. To update stress from the nonlinear Eq. (38), Newton-Raphson is also used. At this level of local iteration, computation of stress $\sigma_{\mathrm{n}+1}$ and the corresponding consistent tangent modulus is performed at each Gauss quadrature point (material point). Such computation at the local level forms the main task of numerical solution procedure and is explained below.

The proposed constitutive model is formulated by the combination of CDM and Drucker-Prager multi-surface plasticity model. The computational algorithm employs operator split method to decompose the constitutive relation in Eq. (5) into three parts: elastic, plastic and damage parts which are handled by, respectively, the elastic predictor, plastic corrector and damage corrector. $^{7,34}$ Generally, backward Euler return mapping scheme is adopted for the elastic predictor and plastic corrector since it is robust, accurate and unconditionally stable. ${ }^{32-35}$ To preserve the quadratic convergent rate, the consistent tangent modulus is used instead of the classical elastoplastic tangent modulus. ${ }^{33}$

The main purpose of a constitutive integration algorithm is to update basic variables $\left\{\sigma, d_{t}, d_{t}, \varepsilon^{p}, \kappa\right\}$ in a way consistent with constitutive model. During this process, the strain history $\varepsilon=\nabla^{s} u$ is assumed to be given. To define the material behavior, the strain history is given by providing a series of strain increments. Constitutive equations given in Section 2 are solved incrementally over a sequence of time steps $\left[t_{n}, t_{n+1}\right]$. The algorithm is to compute $\left\{\sigma_{n+1}, d_{t, n+1}, d_{c, n+1}, \varepsilon_{n+1}^{p}, \kappa_{n+1}\right\}$ that would satisfy the plastic consistency condition (i.e. $F_{1}=0$ and $F_{2}=0$ ). In this study, the algorithm is similar to that proposed by Simo and Hughes. ${ }^{34}$

It is noted that the algorithm herein requires treatment of multisurface plasticity (combination of two Drucker-Prager surfaces) instead of single-surface plasticity (e.g. as in the case of Von Mises model). The main difference lies in the algorithmic treatment of plastic loading by the trial elastic state. Unlike the implementation of loading/unloading conditions for single-surface plasticity, this work for multi-surface plasticity is not straightforward and an iterative procedure is needed ${ }^{34}$. As usual in single surface plasticity, the elastic response is simply characterized by

$$
\sigma=C:\left(\varepsilon-\varepsilon^{p}\right)
$$

The characterization of the elastic domain $E_{\sigma}$ for two-surface plasticity (as shown in Fig. 2) is considerably different from the above and is defined as:

$$
E_{\sigma}=\left\{\left(\bar{\sigma}, \kappa_{t}, \kappa_{c}\right) \mid F_{\alpha}\left(\bar{\sigma}, \kappa_{t}, \kappa_{c}\right) \leq 0, \text { for } \alpha=1,2\right\}
$$

Accordingly, the boundary is:

$$
\partial E_{\sigma}=\left\{\left(\bar{\sigma}, \kappa_{t}, \kappa_{c}\right) \mid F_{\alpha}\left(\bar{\sigma}, \kappa_{t}, \kappa_{c}\right)=0, \text { for } \alpha=1,2\right\}
$$

consisting of two smooth yield surfaces $F_{1}$ and $F_{2}$ which do not join smoothly. Singular points or "corners" are present in the boundary of the elastic domain. Therefore, the evolution of plastic strain is governed by the following Koiter's flow rule ${ }^{36,37}$ :

$$
\dot{\varepsilon}^{p}=\sum_{\alpha=1}^{2} \gamma^{\alpha} \partial_{\bar{\sigma}} F_{\alpha}\left(\bar{\sigma}, \kappa_{t}, \kappa_{c}\right)
$$

Application of an implicit backward Euler scheme to the constitutive equations in section 2 results in the following nonlinear equations for the unknown state $\left(\varepsilon_{n+1}, \varepsilon_{n+1}^{p}, \kappa_{n+1}\right)$ given the set $\left(\varepsilon_{n}, \varepsilon_{n}^{p}, \kappa_{n}\right)$ at time $n$ as the initial conditions:

$$
\begin{aligned}
& \varepsilon_{n+1}=\varepsilon_{n}+\Delta \varepsilon \\
& \bar{\sigma}_{n+1}=C:\left(\varepsilon_{n+1}-\varepsilon_{n+1}^{p}\right) \\
& \varepsilon_{n+1}^{p}=\varepsilon_{n}^{p}+\sum_{\alpha=1}^{2} \Delta \gamma_{n+1}^{\alpha} \partial_{\bar{\sigma}} F_{\alpha}\left(\bar{\sigma}_{n+1}, \kappa_{n+1}\right) \\
& \kappa_{n+1}=\sum_{\alpha=1}^{2} \gamma_{n+1}^{\alpha} H_{\alpha} \\
& F_{\alpha}\left(\bar{\sigma}_{n+1}, \kappa_{n+1}\right)=0, \alpha=1,2
\end{aligned}
$$

The counterpart of the Kuhn-Tucker conditions takes the forms:

$$
\begin{aligned}
& F_{\alpha}\left(\bar{\sigma}_{n+1}, \kappa_{n+1}\right) \leq 0 \\
& \Delta \gamma_{n+1}^{\alpha} \geq 0 \\
& \Delta \gamma_{n+1}^{\alpha} F_{\alpha}\left(\bar{\sigma}_{n+1}, \kappa_{n+1}\right)=0, \alpha=1,2
\end{aligned}
$$

The trial state is obtained by fixing plastic flow in the interval $\left[t_{n}\right.$, $\left.t_{n+1}\right]$ :

$$
\begin{aligned}
& \varepsilon_{n+1}^{p, t \text { trial }}=\varepsilon_{n}^{p} \\
& \kappa_{n+1}^{\text {trial }}=\kappa_{n} \\
& \bar{\sigma}_{n+1}^{\text {trial }}=C:\left(\varepsilon_{n+1}-\varepsilon_{n}^{p}\right) \\
& d_{t, c, n+1}^{\text {trial }}=d_{t, c, n} \\
& F_{\alpha, n+1}^{\text {trial }}=F_{\alpha}\left(\bar{\sigma}_{n+1}^{\text {trial }}, \kappa_{n+1}^{\text {trial }}\right), \alpha=1,2
\end{aligned}
$$

Whether plastic loading or elastic response occurs in the time interval is determined by the following conditions ${ }^{34}$ : 
$F_{\alpha, n+1}^{\text {trial }} \leq 0$ for $\alpha=1$ and $2 \rightarrow$ elastic step

$F_{\alpha, n+1}^{\text {trial }}>0$ for $\alpha=1,2$ or $\alpha=1$ or $\alpha=2 \rightarrow$ plastic step

If only one yield surface is active (i.e. $\alpha=1$ or $\alpha=2$ ), the condition $F_{\alpha, n+1}^{t r i a l}>0$ implies that $\Delta \gamma_{n+1}^{\alpha}>0$. This means the $\alpha$ yield surface is active and this agrees with the conclusion drawn in the single surface plasticity. However, if both of these two yield surfaces are active, the condition $F_{\alpha, n+1}^{\text {trial }}>0$ does not necessarily mean $\Delta \gamma_{n+1}^{\alpha}>0$. In other words, it is possible that $F_{\alpha, n+1}^{\text {trial }}>0$ and, at the same time, $\Delta \gamma_{n+1}^{\alpha}>0$. In Fig. 5 the whole region is divided into four parts and their corresponding stress states are:

Elastic

state:

(1) $F_{1, n+1}^{\text {trial }}<0, F_{2, n+1}^{\text {trial }}<0$

Plastic

state:

(2) $F_{1, n+1}^{\text {trial }}>0, F_{2, n+1}^{\text {trial }}>0\left\{\begin{array}{l}A: \Delta \gamma_{n+1}^{1}>0 \& \Delta \gamma_{n+1}^{2}<0 \\ B: \Delta \gamma_{n+1}^{1}>0 \& \Delta \gamma_{n+1}^{2}>0 \\ C: \Delta \gamma_{n+1}^{1}<0 \& \Delta \gamma_{n+1}^{2}>0\end{array}\right.$

(3) $F_{1, n+1}^{\text {trial }}>0, F_{2, n+1}^{\text {trial }}<0$

(4) $F_{1, n+1}^{\text {trial }}<0, F_{2, n+1}^{\text {trial }}>0$

If the trial stress lies in Region (2) which is divided into three sub-regions according to the conditions shown above, an iterative procedure is needed to determine the set of active constraints defined as $J_{\text {act }}$ from an initial set of trial constraints defined as:

$$
J_{a c t}^{\text {trial }}=\left\{\alpha \in\{1,2\} \mid F_{\alpha, n+1}^{\text {trial }}>0\right\}
$$

Similar to that proposed by Simo and Hughes, ${ }^{34}$ the return mapping algorithm for multi-surface plasticity is derived and summarized in Appendix A. The damage parameter is then calculated from Eq. (9):

$$
D_{n+1}=1-\left(1-d_{t, n+1}\right)\left(1-d_{c, n+1}\right)
$$

and the stress is updated as:

$$
\sigma_{n+1}=\left(1-D_{n+1}\right) \bar{\sigma}_{n+1}
$$

After the stress update, the consistent tangent modulus needs to be computed. The algorithmic modulus for the backward Euler scheme is defined as:

$$
C^{a \lg }=\left(\frac{d \sigma}{d \varepsilon}\right)^{n+1}
$$

For multi-surface plasticity, this would involve the following matrix:

$$
\begin{aligned}
& {[R]=\left\{\begin{array}{l}
d \bar{\sigma}_{n+1} \\
d \kappa_{n+1}
\end{array}\right\}=} \\
& {\left[A_{n+1}-\frac{\left(\sum_{\alpha=1}^{2}\left(A_{n+1}:\left\{\bar{r}_{n+1}^{\alpha}\right\}\right)\right) \otimes\left(\sum_{\alpha=1}^{2}\left(\partial F_{n+1}^{\alpha}: A_{n+1}\right)\right)}{\sum_{\alpha=1}^{2}\left(\partial F_{n+1}^{\alpha}: A_{n+1}:\left\{\bar{r}_{n+1}^{\alpha}\right\}\right)}\right]:\left\{\begin{array}{l}
\left.d \varepsilon_{n+1}\right\} \\
0
\end{array}\right\}}
\end{aligned}
$$

From Eq. (58), we obtain:

$$
\left(\frac{d \bar{\sigma}}{d \varepsilon}\right)^{n+1}=[R]_{n \times n}
$$

To obtain the algorithmic modulus $\left(\frac{d \sigma}{d \varepsilon}\right)^{n+1}$ in Eq. (57), we can differentiate Eq. (56):

$$
d \sigma_{n+1}=\left(1-D_{n+1}\right) \cdot d \bar{\sigma}_{n+1}-d D_{n+1} \cdot \bar{\sigma}_{n+1}
$$

Computation of the algorithmic modulus in this way is exceedingly laborious for complicated plastic-damage models. ${ }^{22,24,34}$ However, the accuracy of definition of the material Jacobian matrix only affects the convergence rate and the results are unaffected. Therefore, an approximate material Jacobain matrix based on the following equation can be used at a slower convergence rate instead of using Eq. (60). ${ }^{38,39}$

$$
\left(\frac{d \sigma}{d \varepsilon}\right)^{n+1}=(1-D)\left(\frac{d \bar{\sigma}}{d \varepsilon}\right)^{n+1}
$$

\section{Results and discussion}

The proposed plastic-damage model has been implemented in an established finite element analysis software called ABAQUS ${ }^{29}$. The numerical results obtained based on the proposed plasticdamage model are compared with the experimental results. Five examples are presented here, covering both static and dynamic tests. In Example 1, the experimental results are obtained from a published paper whereas in the other four examples, they are obtained by our experimental work. Except for the impact tests, all experimental tests were conducted by means of displacement control in order to obtain the post-peak behavior.

\subsection{Example 1-Concrete plates under biaxial loading}

In the first example, the proposed model is used to simulate the well-known biaxial tests carried out experimentally by Kupfer et al. $^{25}$ on plain NWC plates $200 \times 200 \times 50 \mathrm{~mm}$ with grade of 32 $\mathrm{MPa}$. Three different stress ratios $\sigma_{1} / \sigma_{2}$ were applied in the tests: pure compression $\left(\sigma_{1} / \sigma_{2}=-1: 0\right)$; double symmetric compression $\left(\sigma_{1} / \sigma_{2}=-1:-1\right)$; double non-symmetric compression $\left(\sigma_{1} /\right.$

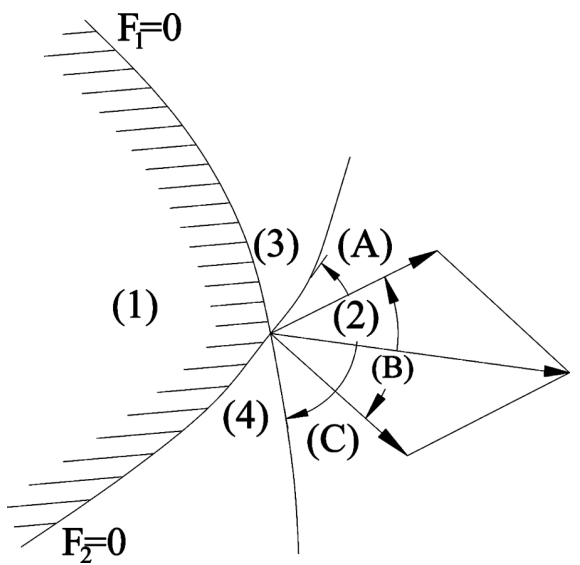

Fig. 5 Illustration of geometry at intersection point of two yield surfaces. 
$\left.\sigma_{2}=-1:-0.52\right)$. In these three cases, $\sigma_{3}$ is zero. Due to symmetry in the $x$ and $y$ planes, only one quarter of the plate is modeled in the numerical simulation. The biaxial compressive loads are applied on the remaining free surfaces as illustrated in Fig. 6. In the FE model, 4-node bilinear, reduced integration plain stress elements are adopted. A total of 16 elements of size $25 \times 25 \mathrm{~mm}$ are used in this example. The material parameters are shown in Table 1 . In addition, the uniaxial stress-strain curve obtained by the experiment is used as the input.

The numerical and experimental stress-strain curves for pure compression are compared in Fig. 7. The comparisons for double symmetric compression and double non-symmetric compression are presented in Figs. 8 and 9, respectively. In all three cases of comparison, the numerical results obtained by the proposed model are in very good agreement with the experimental results. These results also show that the compressive strength of biaxial compression is dependent on stress ratio $\sigma_{1} / \sigma_{2}$, i.e. the compressive strength of biaxial compression is improved compared to that of uniaxial compression. Thus, the proposed model has the ability to reflect correctly the concrete strength enhancement under biaxial compression.

\subsection{Example 2-Concrete cylinders under uniaxial loading}

In the second example, we carried out experimental investigation on LWC and NWC cylinders under uniaxial loading. ASTM Type I ordinary Portland cement was used for making all the specimens. For LWC, the light weight aggregates used in this study are lightweight expanded clay aggregate (LECA) with bulk density $800 \mathrm{~kg} / \mathrm{m}^{3}$. For NWC, crushed granite aggregates with a specific gravity of 2.70 and a maximum nominal size of $20 \mathrm{~mm}$ were used. River sand with a specific gravity of 2.7 was used and the grading of the sand meets the requirements of ASTM C33 as well. Superplasticizer was used to improve the workability when needed. NWC mix with design strength of $50 \mathrm{MPa}$ was used as a benchmark specimen. The LWC mixes were designed to have different strengths, i.e. $40 \mathrm{MPa}, 60 \mathrm{MPa}$ and $80 \mathrm{MPa}$. The four mixes are

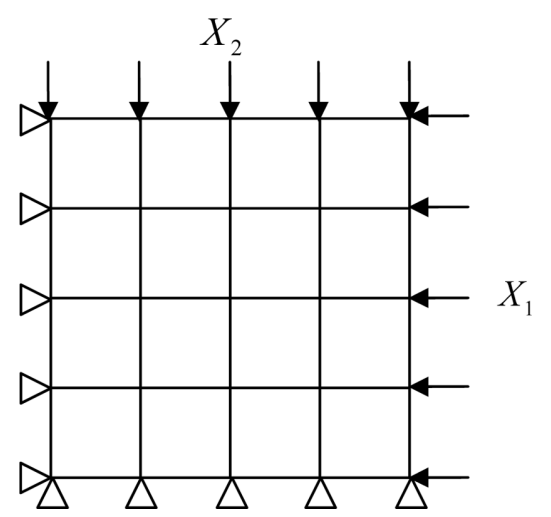

Fig. 6 Finite element model of NWC plate tested by Kupfer, et al. $^{25}$ (Example 1).

Table 1 Material parameters used in Example 1

\begin{tabular}{c|c}
\hline Young's modulus $E(\mathrm{GPa})$ & 30 \\
\hline \hline Poisson's ratio $v$ & 0.2 \\
\hline Compressive strength $f_{c}(\mathrm{MPa})$ & 32 \\
\hline Tensile strength $f_{t}(\mathrm{MPa})$ & 3.2 \\
\hline
\end{tabular}

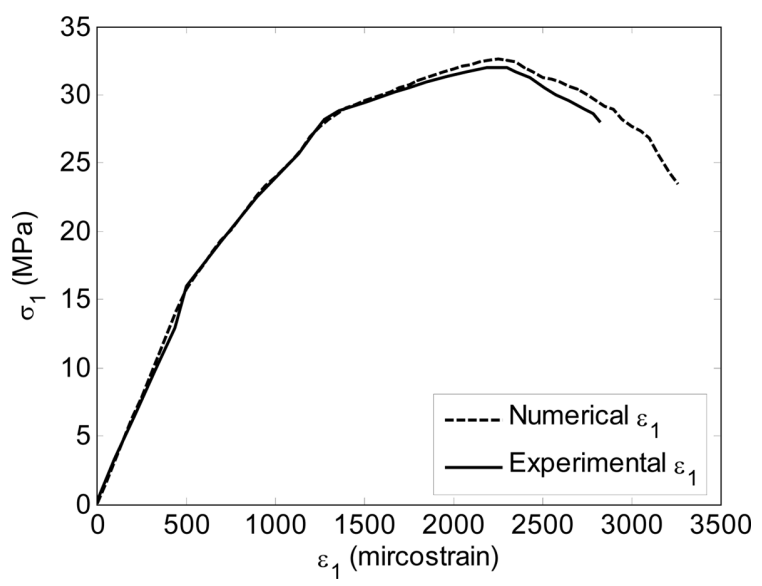

Fig. 7 Stress-strain curves for NWC plate under $-1: 0$ biaxial compression (Example 1).

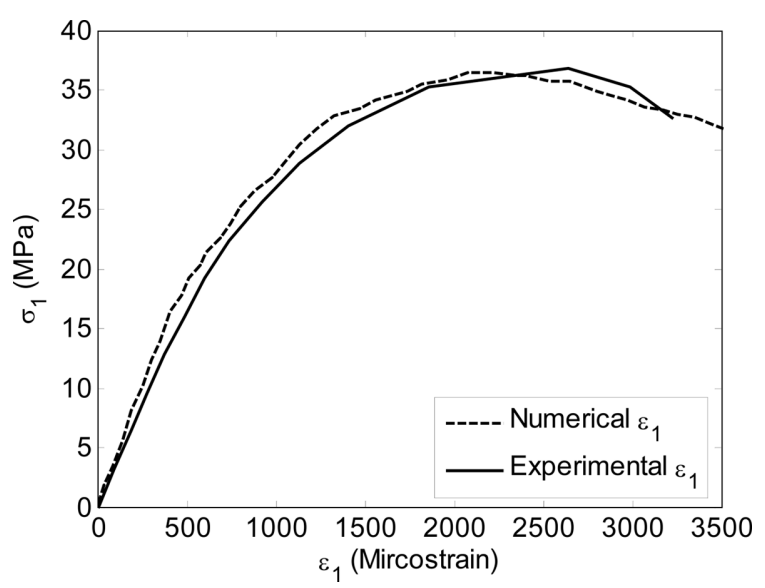

Fig. 8 Stress-strain curves for NWC plate under -1:-1 biaxial compression (Example 1).

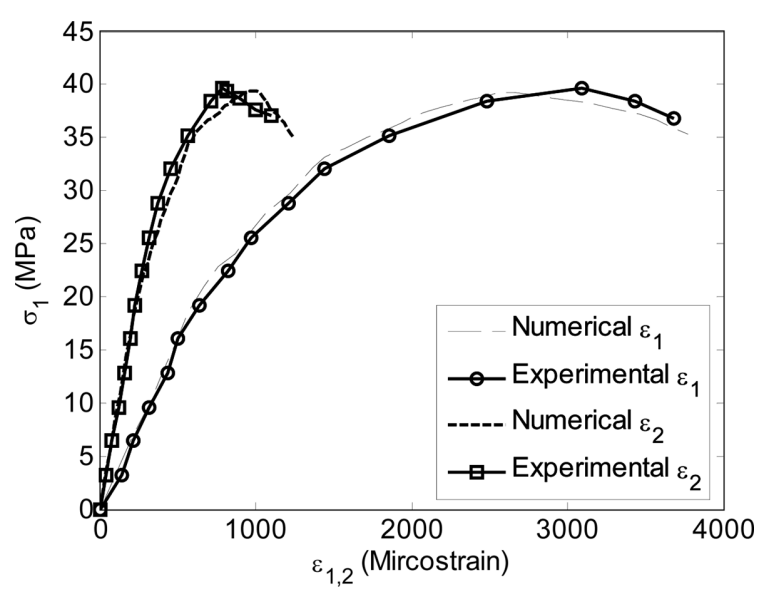

Fig. 9 Stress-strain curves for NWC plate under $-1:-0.52$ biaxial compression (Example 1).

labeled as NWC, LECA40, LECA60 and LECA80, respectively. All the specimens were wet-cured for 28 days before tests were carried out. Static tests were conducted for cylinders to obtain its compressive stress-strain curves (Fig. 10), from which the material parameters such as Young's modulus and Poisson's ratio can be obtained. The same material parameters are used in Examples 3 to 5 to follow. 
In the numerical simulation, concrete cylinders under uniaxial compressive loads are modeled by asymmetric elements since the geometries and the loads are asymmetric as shown in Fig. 11. Figs. 12 to 15 present the numerical and experimental results of uniaxial stress-strain relation of NWC, LECA40, LECA60 and LECA80, respectively. The very good comparison illustrates the applicability of the proposed model under static conditions. These results also show that LWC has a more linear ascending curve indicating that there are few cracks prior to peak stress point as compared to NWC. The proposed mode is shown to be able to capture the different behaviors of NWC as well as LWC with different strengths.

\subsection{Example 3 - Cylinder splitting}

Cylinder splitting test is often used as an indirect way to determine the tensile strength of concrete. As shown in Fig. 16, the splitting test is carried out on a standard cylinder tested on its side in diametrical compression. The load caused the cylinder to split in two halves. The specimens used in the test were of length of $200 \mathrm{~mm}$ and radius of $50 \mathrm{~mm}$. Again due to symmetry, only a quarter of the specimen is needed in the FE model as shown in Fig. 17 involving 160 4-node reduced integration linear plain

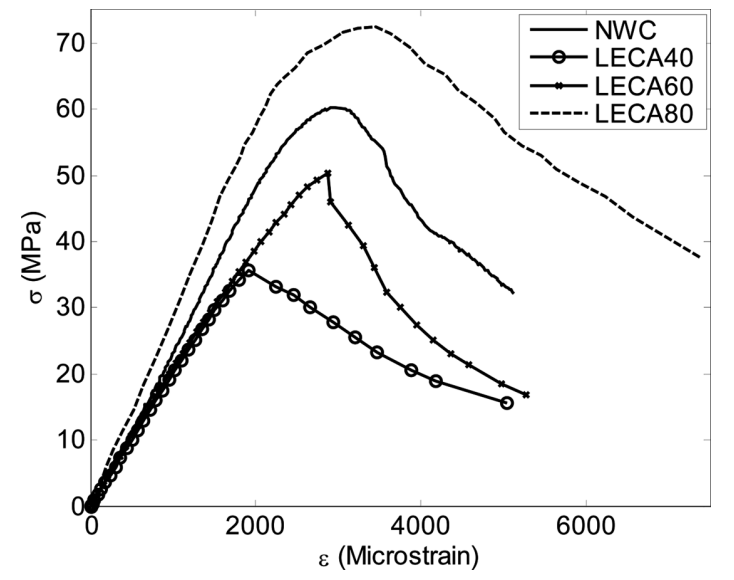

Fig. 10 Stress-strain curves for NWC, LECA40, LECA60 and LECA80 used in Examples 2 to 5.

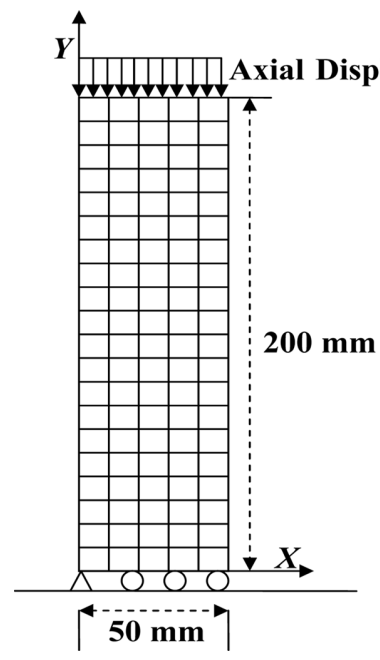

Fig. 11 Finite element Model for cylinder subjected axial loadings (Example 2).

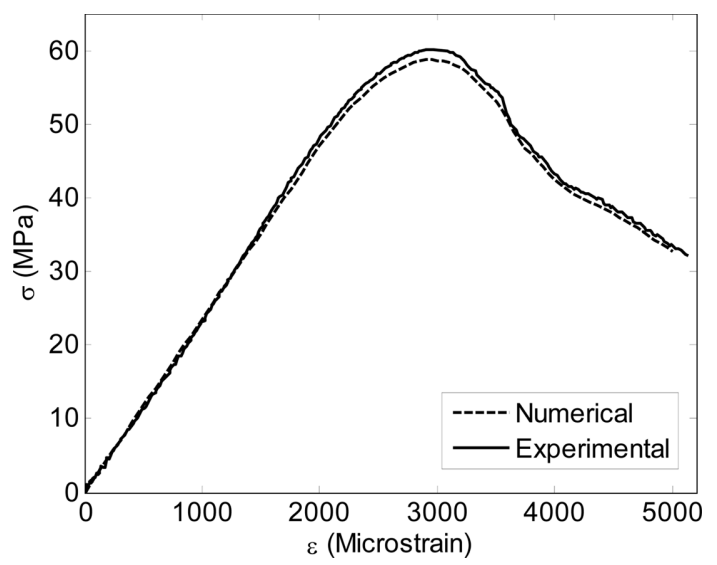

Fig. 12 Stress-strain curve of NWC under uniaxial load (Example 2).

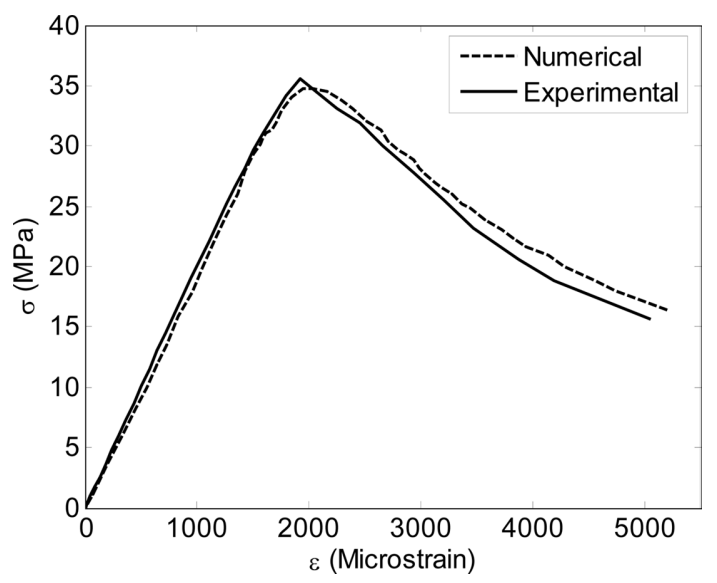

Fig. 13 Stress-strain curve of LECA40 under uniaxial load (Example 2).

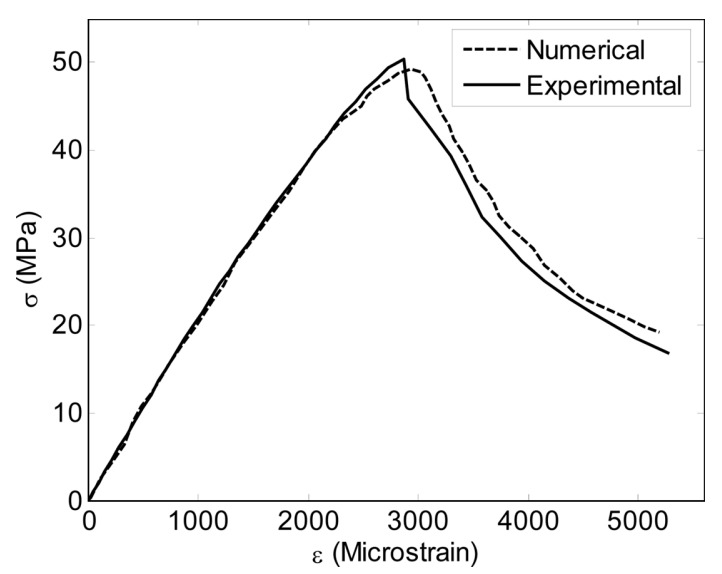

Fig. 14 Stress-strain curve of LECA60 under uniaxial load (Example 2).

stress elements. The load versus displacement at the loading point is depicted in Fig. 18 for all the four NWC and LWC specimens. The tensile strength is calculated by

$$
f_{t}=\frac{2 P}{\pi d L}
$$

where $P$ is the load at which the cylinder failed, $d$ is the cylinder 


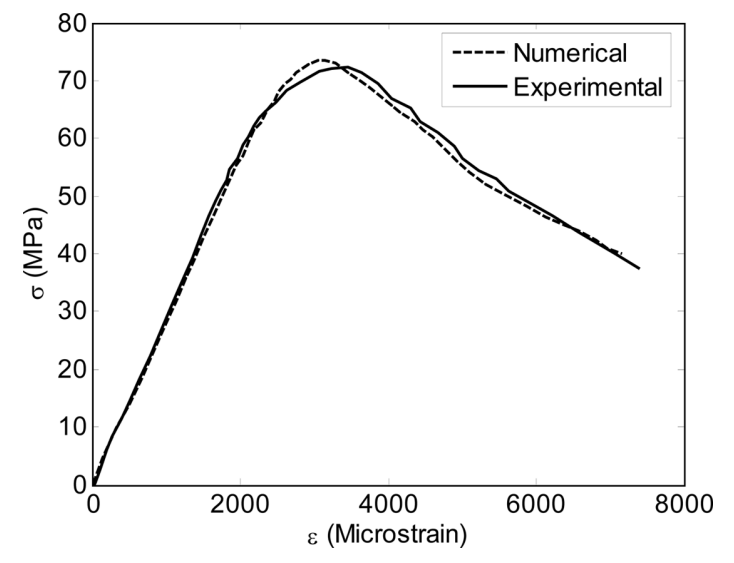

Fig. 15 Stress-strain curve of LECA80 under uniaxial load (Example 2).

diameter and $L$ is the cylinder length.

Figures 19 and 20 show the comparison between experimental and numerical results of failure load and tensile strength, respectively. The comparison shows that the proposed model is again very good in reproducing the splitting test result with error less than $8 \%$ only.

\subsection{Example 4- Notched beams under bending}

In this example, a notched beam is centrally loaded till failure

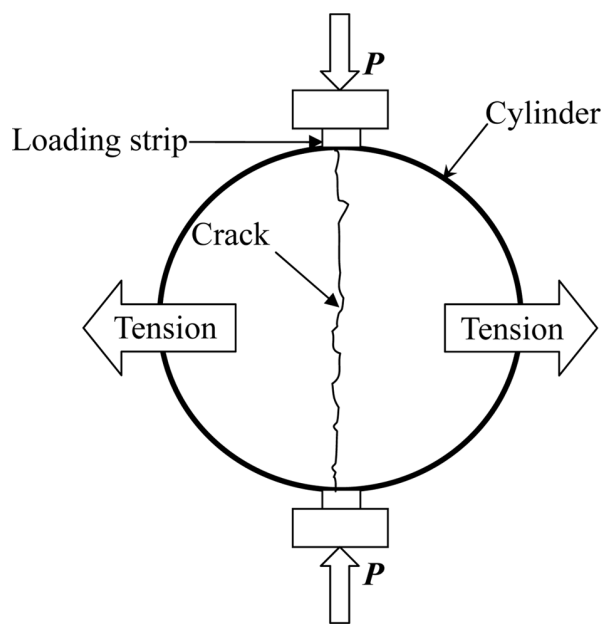

Fig. 16 Cylinder splitting test (Example 3).

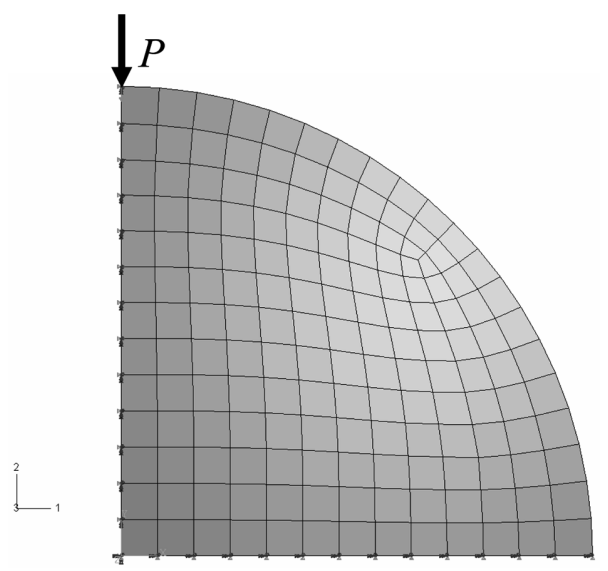

Fig. 17 Finite element model for cylinder splitting test (Example 3). under three-point bending, with a span length of $700 \mathrm{~mm}$, initial notch depth $a_{0}=50 \mathrm{~mm}$ at a constant rate of $0.012 \mathrm{~mm}$ per minute. The experimental set-up is shown in with displacement measured at the midspan, and a schematic diagram of specimen under test is shown in Fig. 22. Notched plain concrete beams for NWC and three LECA specimens are subjected to the displacement-controlled test using the proposed model. Two-dimensional plane stress elements are used to model the specimen and the mesh of the specimen as shown in Fig. 23.

In Figs. 24 to 27, the numerical results for load-displacement

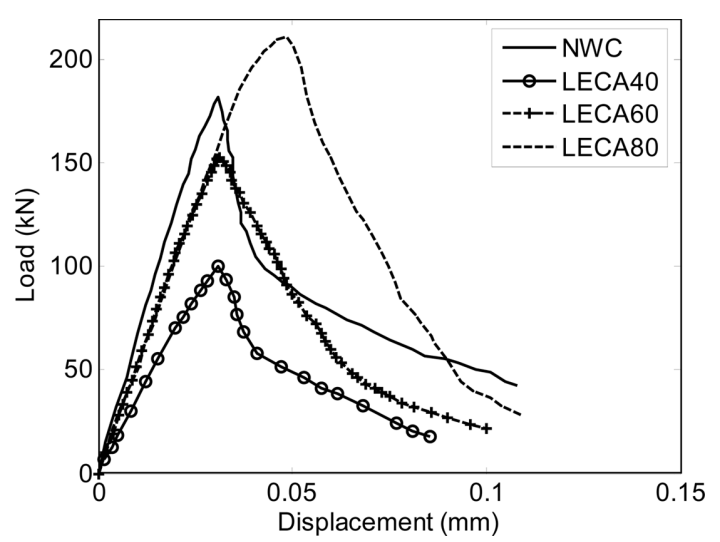

Fig. 18 Numerical results for cylinder splitting test (Example 3).

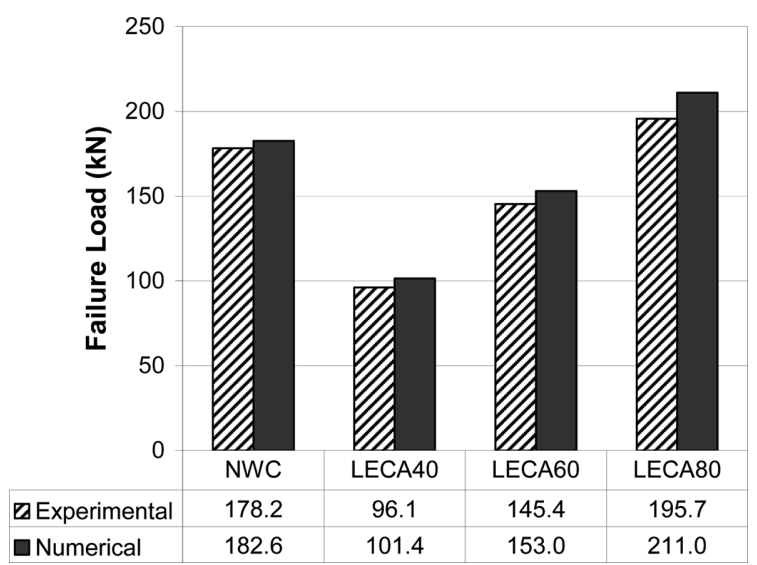

Fig. 19 Experimental and numerical results of failure loads (Example 3).

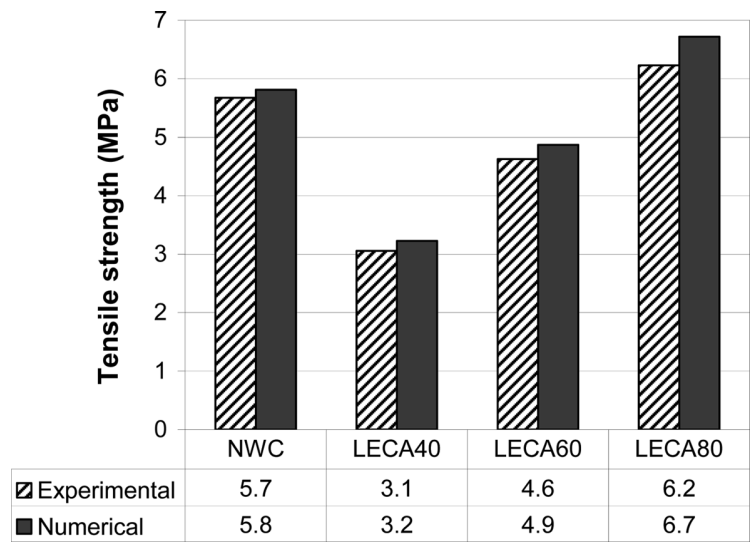

Fig. 20 Experimental and numerical results of tensile strength (Example 3). 


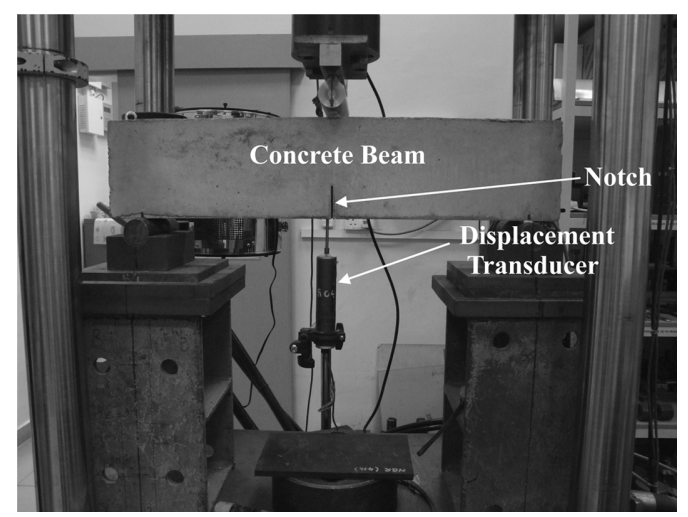

Fig. 21 Bending test of notched beam using a $50 \mathrm{kN}$ INSTRON machine (Example 4).

curves are with the corresponding experimental results for the four concrete types studied. It is evident that the agreement is generally very good particularly before the load approaches the peak load. The agreement for peak load is excellent with error less than $8 \%$. The post-peak agreement is also good in view of the severe nonlinearity after the peak load and the large damage accumulated in the beams. The results demonstrate the capability of the proposed model for both NWC and LWC considered involving crack propagation from the notch.

\subsection{Example 5 - Slab under impact loading}

In the final example, impact test of concrete slab by drop-weight experiments were conducted as shown in Fig. 28. The four edges of the slab were fixed by angle plates and G-clampers, and the impactor fell freely from a height of $2 \mathrm{~m}$ above the slab. The cylindrical impactor was a hemisherical-nose steel cylinder of diameter $100 \mathrm{~mm}$ and mass $47 \mathrm{~kg}$. The impactor was lifted by a suspending steel wire and a winch to the intended height. It was then dropped suddenly inside a guiding tube by releasing the winch. The velocity of impactor before hitting the slab was measured by a laser diode system. Two displacement transducers measured the deformations on the bottom side of the slab at points $\mathrm{P}_{1}$ and $\mathrm{P}_{2}$ as indicated in Fig. 29.

By symmetry, only one quarter of the slab and the impactor are

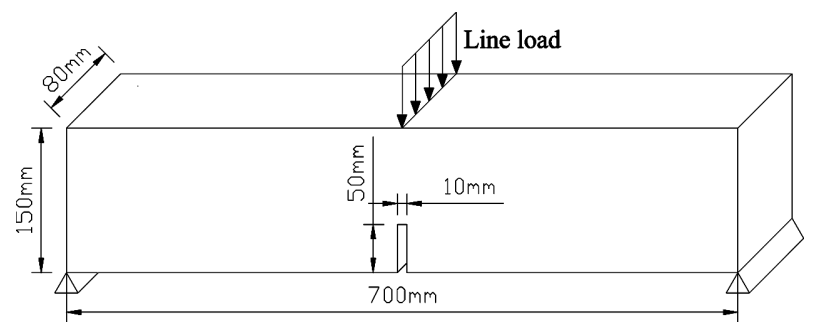

Fig. 22 Dimensions of notched beam (Example 4).

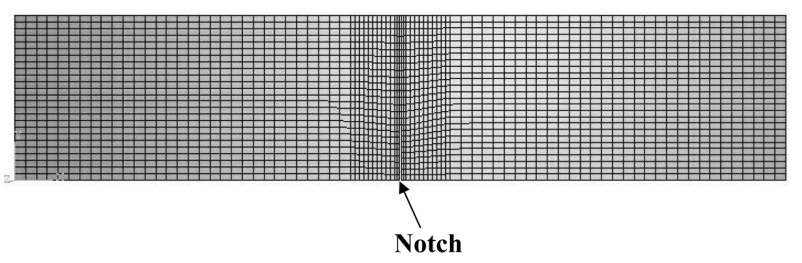

Fig. 23 FE Mesh of the notched beam (Example 4).

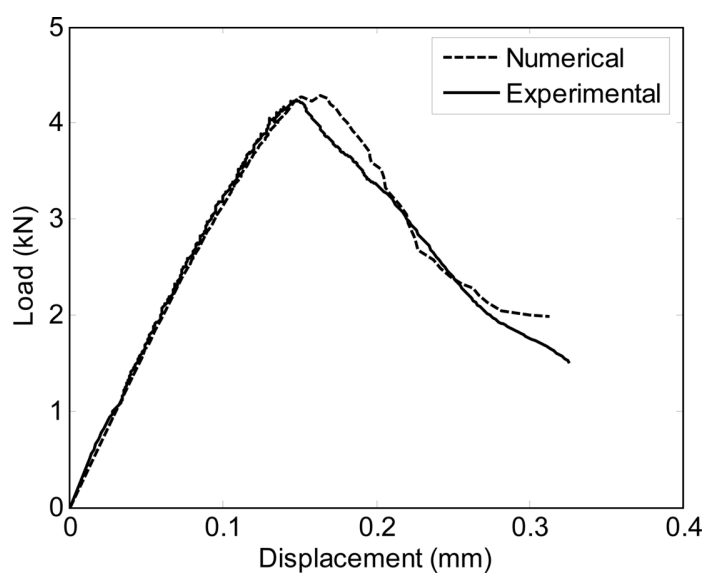

Fig. 24 Experimental and numerical results for NWC notched beam (Example 4).

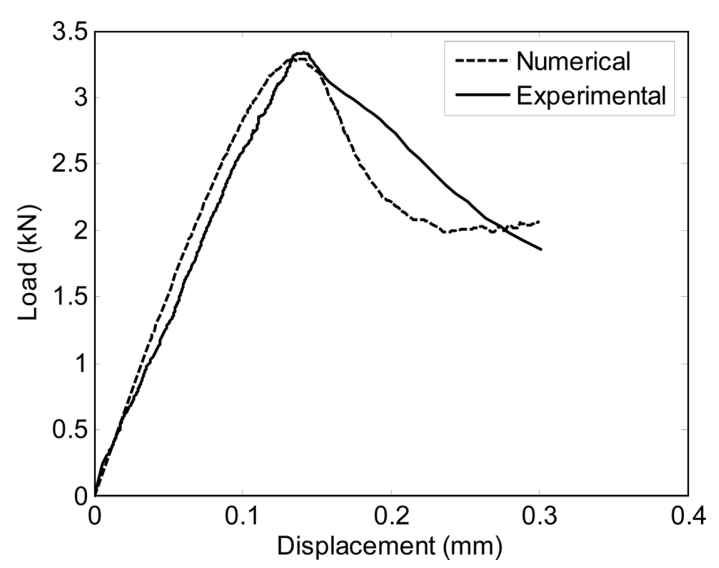

Fig. 25 Experimental and numerical results for LECA40 notched beam (Example 4).

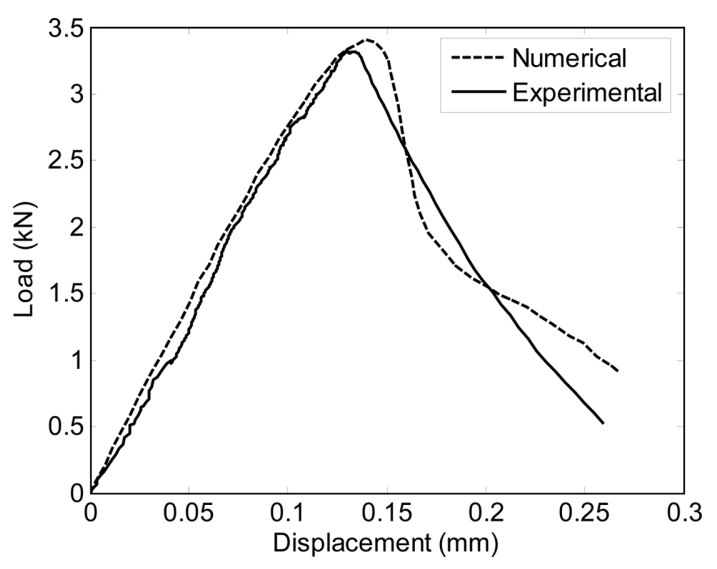

Fig. 26 Experimental and numerical results for LECA60 notched beam (Example 4).

considered in the FE model (Fig. 30). The impactor is modeled by 3008 -node linear reduced-integration solid elements, and the slab by 8-node linear reduced-integration solid elements with mesh size $10 \mathrm{~mm}$. The reinforcing bars in the slab are models as onedirectional strain elements and are simulated by using the REBAR option in $\mathrm{ABAQUS}{ }^{29}$ that uses a superposition method whereby the reinforcing bars are superposed on a mesh of finite element for concrete.

For the four NWC and LWC slabs under impact, Figs. 31 to 34 


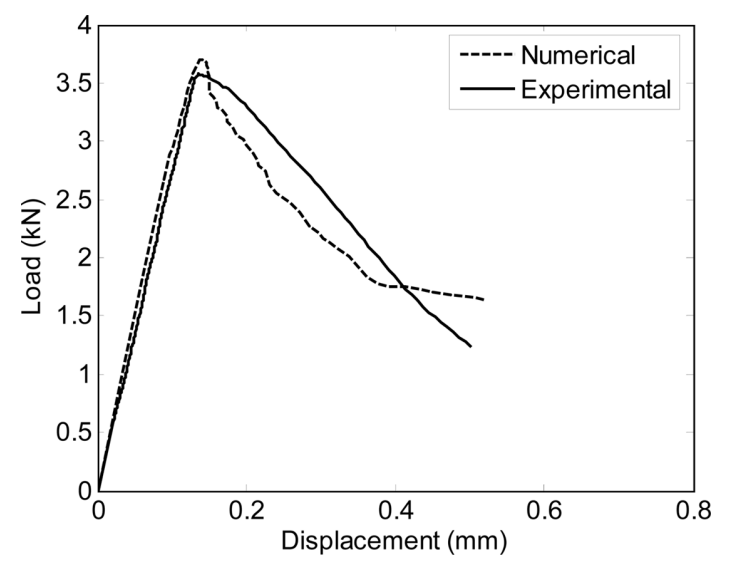

Fig. 27 Experimental and numerical results for LECA80 notched beam (Example 4).

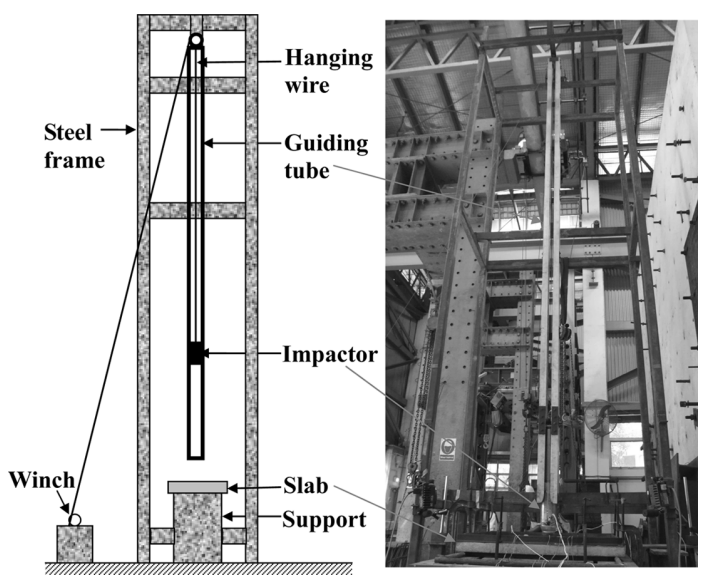

Fig. 28 Impact test set-up (Example 5).

present the FE results for the deformation time histories at $\mathrm{P}_{1}$ (the same for $\mathrm{P}_{2}$ due to symmetry). For the experimental results, the average value of measured deformations at $\mathrm{P}_{1}$ and $\mathrm{P}_{2}$ is used. It is seen that the numerical results slightly overestimate the maximum deformation of the point of the slab but the error is only less than $10 \%$. The post-peak curves in the numerical results exhibit steeper slopes than the experimental results. This could be due to the internal frictional resistance of the displacement transducer that caused

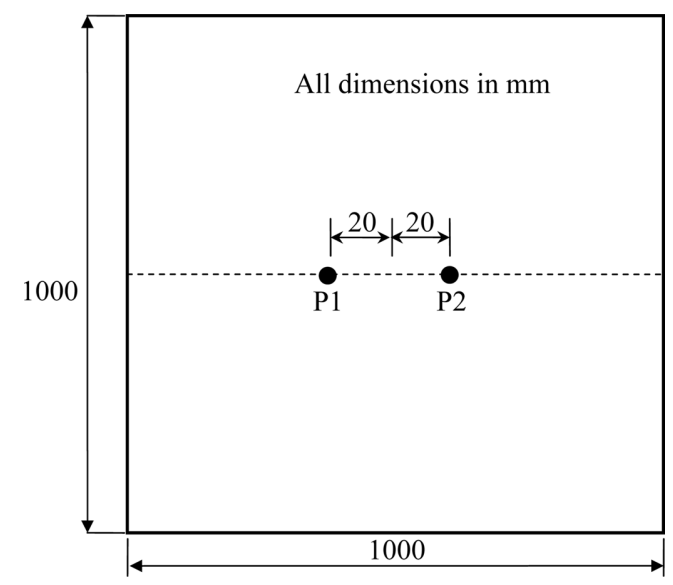

Fig. 29 Locations of displacement measurement on the bottom of the slab (Example 5). a slight time delay between the actual and recorded deformation. On the whole, the numerical results generally correlate very well with the experimental results, validating the applicability of the proposed model for both NWC and LWC under impact loadings.

\section{Conclusions}

This paper presents a new plastic-damage constitutive model for LWC and NWC within the theoretical framework of both CDM and plasticity. Two damage variables are used to represent tensile

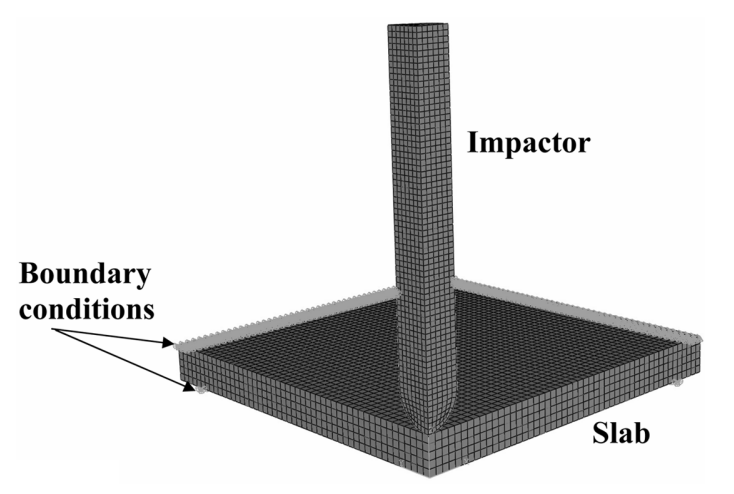

Fig. $30 \mathrm{FE}$ model of one quarter of slab and impactor (Example 5).

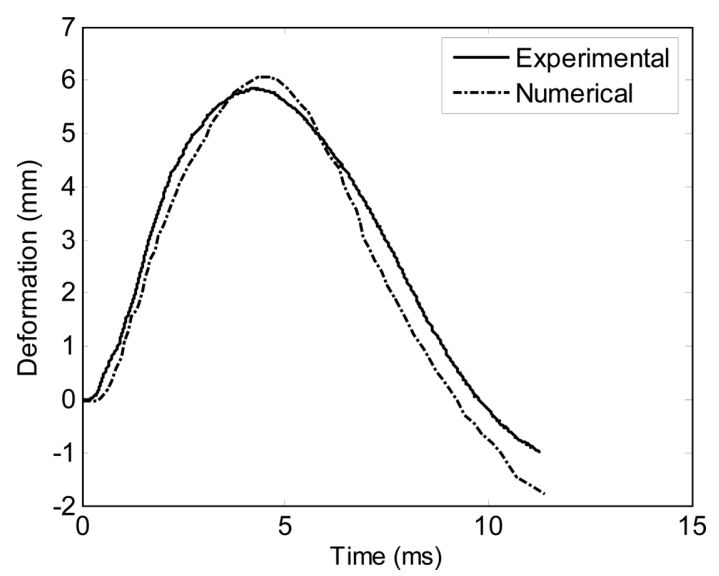

Fig. 31 Experimental and numerical results for NWC slab under impact load (Example 5).

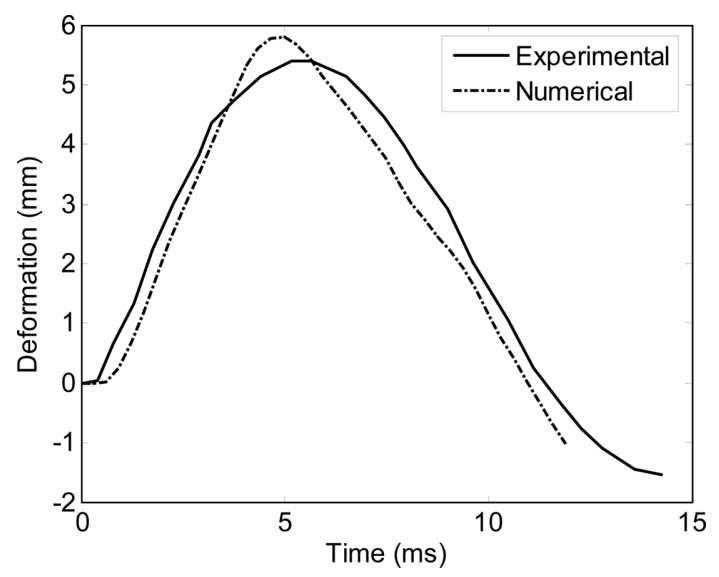

Fig. 32 Experimental and numerical results for LECA40 slab under impact load (Example 5). 


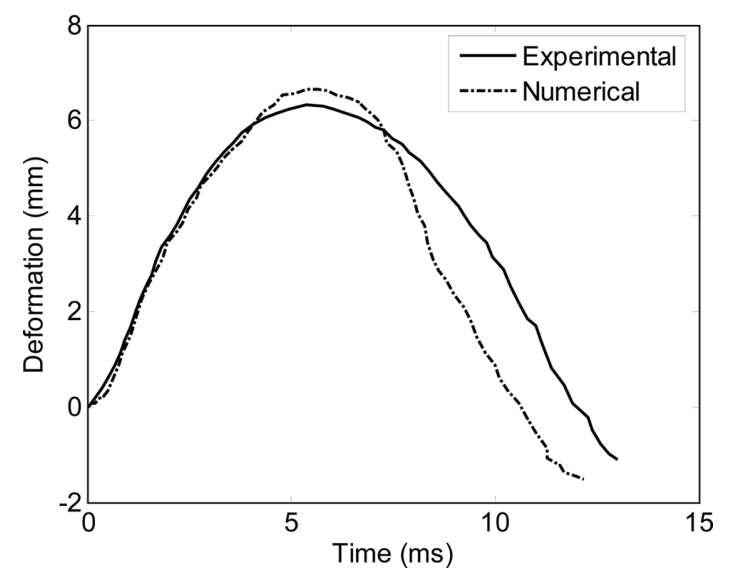

Fig. 33 Experimental and numerical results for LECA60 slab under impact load (Example 5).

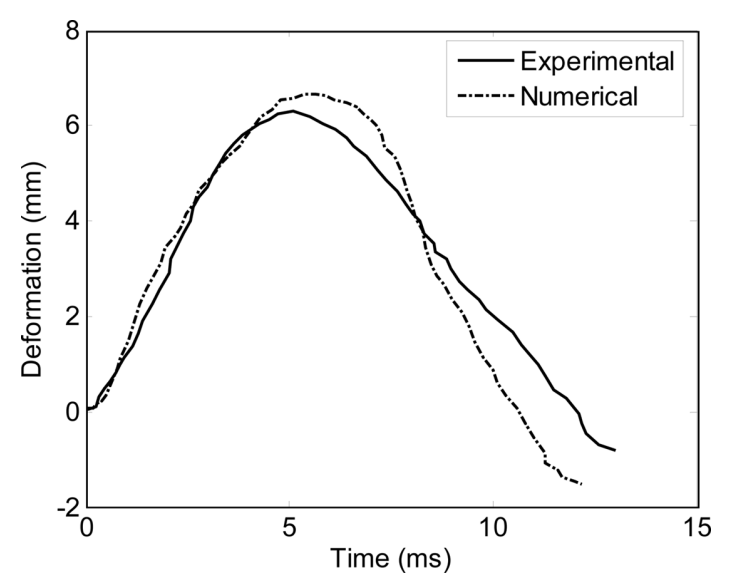

Fig. 34 Experimental and numerical results for LECA80 slab under impact load (Example 5).

Table 2 Material parameters used in Examples 2 to 5

\begin{tabular}{c|c|c|c|c}
\hline & NWC & LECA40 & LECA60 & LECA80 \\
\hline \hline Young's modulus, $E(\mathrm{GPa})$ & 23.4 & 19.9 & 21.4 & 29.4 \\
\hline Poisson's ratio $v$ & 0.2 & 0.18 & 0.19 & 0.19 \\
\hline Compressive strength, $f_{c}(\mathrm{MPa})$ & 60.1 & 35.6 & 50.4 & 72.4 \\
\hline Tensile strength, $f_{t}(\mathrm{MPa})$ & 5.8 & 3.2 & 4.9 & 6.7 \\
\hline
\end{tabular}

and compressive damage, respectively, whereas the effective stress is computed by multi-surface plasticity Drucker-Prager model. The stress is updated by multiplication of the damaged part and the effective part. The proposed model is coded as a user material subroutine and incorporated successfully in a finite element analysis software. Extensive experimental investigation was carried out on LWC and NWC to verify the proposed model. Published experimental results for NWC specimens were also used. The very good comparison between the numerical and experimental results convincingly demonstrates that the proposed constitutive model is able to accurately capture the damage behavior of LWC and NWC under both static and dynamic loading conditions.

\section{References}

1. Clark, J. L., Structural Lightweight Aggregate Concrete, Blackie Academic and Professional, London, 1993.
2. ACI Committee 213, Guide for Structural Lightweight Aggregate Concrete (ACI 213R-03), American Concrete Institute, Farmington Hills, Michigan, 2003.

3. Bathe, K. J., Finite Element Procedures, Prentice Hall Englewood Cliffs, New Jersey, 1996.

4. Wu, C. H., "Tension Compression Test of a Concrete Specimen via a Structure Damage Theory," Proceedings of Continuum Damage Theory and Effective Moduli and Continuum Modeling of Discrete Structure, ASCE, New York, 1985, pp.1 12.

5. Krajcinovic, D. and Fanella, D., "A Micromechanical Damage Model for Concrete," Engineering Fracture Mechanics, Vol.25, No.5-6, 1986, pp. 585 596.

6. Sumarac, D. and Krajcinovic, D., "A Self-consistent Model for Microcrack-weakened Solid," Mechanics of Materials, Vol.6, No.4, 1987, pp. 39 52.

7. Ju, J. W., "On Energy-based Coupled Elastoplastic Damage Theories: Constitutive Modeling and Computational Aspects," International Journal of Solids and Structures, Vol.25, No.7, 1989, pp. 803 833.

8. Ohtani, Y. and Chen, W. F., "Multiple Hardening Plasticity for Concrete Materials," Journal of Engineering Mechanics, ASCE, Vol.114, 1988, pp. 1890 1910.

9. Feenstra, P. H. and De Borst, R., "A Composite Plasticity Model for Concrete," International Journal of Solids and Structures, Vol.33, No.5, 1996, pp. 707 730.

10. Chen, W. F., Constitutive Equations for Engineering Materials, Elsevier, London, 1994.

11. Griffith, A. A., "The Theory of Rupture," Proceedings of the $1^{\text {st }}$ Institutional Congress of Applied Mechanics, Delft, 1925, pp.55 63.

12. Bazant, Z. P. and Planas, J., Fracture and Size Effect in Concrete and Other Quasibrittle Materials, CRC Press, Boca Raton, Florida, 1999.

13. Elfgren, L., ed, Fracture mechanics of concrete structures, Chapman and Hall, London, 1989.

14. ACI Committee 446, Fracture Mechanics of Concrete: Concept, Models, and Determination of Material Properties, American Concrete Institute, 1992.

15. Mazars, J. and Pijaudier-Cabot, G., "Continuum Damage Theory-Application to Concrete," Journal of Engineering Mechanics, ASCE, Vol.115, No.2, 1989, pp. 345 365.

16. Cervera, M., Oliver, J., and Faria, R., "Seismic Evaluation of Concrete Dams via Continuum Damage Models," Earthquake Engineering and Structural Dynamics, Vol.24, No.9, 1995, pp. $1225 \sim 1245$.

17. Mazars, J., "A Model of Unilateral Elastic Damageable Material and Its Application to Concrete," Proceedings of the RILEM International Conference on Fracture Mechanics of Concrete, Lausanne, Switzerland, 1985, pp.61 71.

18. Ortiz, M., "A Constitutive Theory for the Inelastic Behavior of Concrete," Mechanics of Materials, Vol.4, No.1, 1985, pp. 67 93.

19. Chow, C. L. and Wang, J., "An Anisotropic Theory of Continuum Damage Mechanics for Ductile Fracture," Engineering Fracture Mechanics, Vol.27, No.5, 1987, pp. 547 558.

20. Simo, J. C. and Ju, J. W., "Strain- and Stress-based Continuum Damage Models-I. Formulation," International Journal of Solids and Structures, Vol.23, No.7, 1987a, pp. 821 840. 
21. Lemaitre, J., "A Continuum Damage Mechanics Model for Ductile Fracture," Journal of Engineering Materials and Technology, Vol.107, No.1, 1985, pp. 83 89.

22. Lee, J. and Fenves, G. L., "Plastic-Damage Model for Cyclic Loading of Concrete Structures," Journal of Engineering Mechanics, ASCE, Vol.124, No.8, 1998, pp. 892 900.

23. Yazdani, S. and Schreyer, H. L., "Combined Plasticity and Damage Mechanics Model for Plain Concrete," Journal of Engineering Mechanics, ASCE, Vol.116, No.7, 1990, pp. 1435 1450.

24. Lubliner, J., Oliver, J., Oller, S., and Onate, E., "A Plasticdamage Model for Concrete," International Journal of Solids and Structures, Vol.25, No.3, 1989, pp. 299 326.

25. Kupfer, H., Hilsdorf, H. K., and Rusch, H., "Behaviour of Concrete under Biaxial Stress," Journal of American Concrete Institute, Vol.66, No.8, 1969, pp. 656 666.

26. Lee, J. and Fenves, G. L., "A Plastic-damage Concrete Model for Earthquake Analysis of Dams," Earthquake Engineering and Structural Dynamics, Vol.27, No.9, 1998, pp. 937 956.

27. Wu, J. Y., Li, J., and Faria, R., “An Energy Release Ratebased Plastic-damage Model for Concrete," International Journal of Solids and Structures, Vol.43, No.3-4, 2006, pp. 583 612.

28. Koiter, W. T., "General Theorems for Elastic-plastic Solids," Progress in Solid Mechanics 6, Amsterdam, Netherland, 1960, pp.127 221.

29 ABAQUS Theory manual, Version 6.1, Hibbitt, Karlsson and Sorensen Inc, 2001.

30. Hughes, T. J. R., The Finite Element Method: Linear Static and Dynamic Finite Element Analysis, Prentice-Hall, Englewood Cliffs, New Jersey, 1987.

31. Zienkiewicz, O. C. and Taylor, R. L., The Finite Element Method, V.1. The Basis, Butterworth Heinemann, Oxford, 2000.

32. Belytschko, T., Liu, W. K., and Moran, B., Nonlinear Finite Elements for Continua and Structures, John Wiley and Sons, New York, 2000.

33. Simo, J. C. and Taylor, R. L., "Consistent Tangent Operators for Rate-independent Elastoplasticity," Computer Methods in Applied Mechanics and Engineering, Vol.48, No.1, 1985, pp. 101 118.

34. Simo, J. C. and Hughes, T. J. R., Computational Inelasticity, Springer, New York, 1998.

35. Simo, J. C. and Hughes, T. J. R., "On the Variational Foundations of Assumed Strain Methods," Journal of Applied Mechanics, Vol.53, No.1, 1986, pp. 51 54.

36. Koiter, W. T., "Stress-Strain Relations, Uniqueness and Variational Theorems for Elastic-Plastic Materials with a Singular Yield Surface," Quarterly of Applied Mathematics, Vol.11, No.2, 1953, pp. $350 \sim 354$.

37. Naghdi, P. M., "Stress-Strain Relations in Plasticity and Thermoplasticity," Proceedings of the $2^{\text {nd }}$ Symposium on Naval Structural Mechanics, Pergamon Press, London, 1960, pp.121 169.

38. ABAQUS/Standard User's Manual, Version 6.1, Hibbitt, Karlsson and Sorensen Inc, 2001.

39. Blaheta, R., "Convergence of Newton-type Methods in Incremental Return Mapping Analysis of Elasto-plastic Problems," Computer Methods in Applied Mechanics and Engineering, Vol.147, No.1-2, 1997, pp. 167 185.

\section{Appendix A: Return mapping algorithm for multi-surface plasticity}

1. Set initial values: $\bar{\sigma}_{n+1}=\bar{\sigma}_{n+1}^{t r i a l}, \kappa_{n+1}=\kappa_{n}, \varepsilon_{n+1}^{p}=\varepsilon_{n}^{p}, \Delta \gamma^{\alpha}=0, \alpha=1,2$

2. Computer elastic predictor

$\bar{\sigma}_{n+1}^{\text {trial }}=C:\left(\varepsilon_{n+1}-\varepsilon_{n}^{p}\right)$

$F_{\alpha, n+1}^{\text {trial }}=F_{\alpha}\left(\bar{\sigma}_{n+1}^{\text {trial }}, K_{n+1}^{\text {trial }}\right), \alpha=1,2$

3. Check the current yielding state

If $F_{\alpha, n+1}^{\text {trial }} \leq 0$ for $\alpha=1$ and 2 then:

Set $(.)_{n+1}=(.)_{n+1}^{\text {trial }}$ and exit.

Else:

$J_{a c t}^{(0)}=\left\{\alpha=1,2 \mid F_{\alpha, n+1}^{\text {trial }} \geq 0\right\}$

$\varepsilon_{n+1}^{p^{(0)}}=\varepsilon_{n}^{p}$

$\Delta \gamma^{\alpha(0)}=0$

Endif

4. Calculate the residuals

$\bar{\sigma}_{n+1}^{(k)}=C:\left(\varepsilon_{n+1}-\varepsilon_{n+1}^{p(k)}\right)$

$R_{n+1}^{(k)}=\left\{\begin{array}{c}-\varepsilon_{n+1}^{p^{(k)}}+\varepsilon_{n}^{p} \\ -\kappa_{n+1}^{p^{(k)}}+\kappa_{n}^{p}\end{array}\right\}+\sum_{\beta \in J_{a c t}^{(k)}} \Delta \gamma^{\beta(k)}\left\{\begin{array}{c}\partial_{\bar{\sigma}} F_{\beta} \\ \partial_{\kappa} F_{\beta}\end{array}\right\}_{n+1}^{(k)}$

5. Check convergence

If $F_{\alpha, n+1}^{(k)} \leq T O L 1$ for all $\alpha \in J_{a c t}^{(k)}$ and $\left\|R_{n+1}^{(k)}\right\|<T O L 2$, then

Exit

End if

6. Computer increment in plasticity parameter:

$\left\{\begin{array}{l}\delta \gamma^{1} \\ \delta \gamma^{2}\end{array}\right\}=\left[\begin{array}{ll}r^{1,(k)}: A: r^{1,(k)} & r^{1,(k)}: A: r^{2,(k)} \\ r^{2,(k)}: A: r^{1,(k)} & r^{2,(k)}: A: r^{2,(k)}\end{array}\right]^{-1}\left\{\begin{array}{l}F_{1}-F_{1, \bar{\sigma}} A^{(k)} R^{(k)} \\ F_{2}-F_{2, \bar{\sigma}} A^{(k)} R^{(k)}\end{array}\right\}$

$\Delta \bar{\gamma}^{\alpha(k+1)}=\Delta \gamma^{\alpha(k)}+\delta \gamma^{\alpha(k)}$

If: $\Delta \bar{\gamma}^{\alpha(k+1)}<0, \alpha \in J_{a c t}^{(k)}$, then

$$
\text { Update } J_{\text {act }}^{(k+1)}=\left\{\alpha \in J_{\text {act }}^{(k)} \mid \Delta \bar{\gamma}^{\alpha(k+1)}>0\right\}
$$

Go to 4

Endif

7. Obtain incremental plastic strains and hardening parameters:

$$
\begin{aligned}
& \Delta \bar{\sigma}_{n+1}^{(k)}=A_{n+1}^{(k)}:\left[-R_{n+1}^{(k)}-\sum_{\alpha=1}^{2} \delta \gamma_{n+1}^{\alpha(k)} r^{\alpha}\right] \\
& \Delta \varepsilon_{n+1}^{p(k)}=-C^{-1}: \Delta \bar{\sigma}_{n+1}^{(k)}
\end{aligned}
$$

8. Update the plastic strains, hardening parameters and plastic consistent parameters:

$$
\begin{aligned}
& \varepsilon_{n+1}^{p(k+1)}=\varepsilon_{n+1}^{p(k)}+\Delta \varepsilon_{n+1}^{p(k)}=\varepsilon_{n+1}^{p(k)}-C^{-1}: \Delta \bar{\sigma}^{(k)} \\
& \kappa_{n+1}^{(k+1)}=\kappa_{n+1}^{(k)}+\Delta \kappa_{n+1}^{(k)} \\
& \Delta \gamma_{n+1}^{\alpha(k+1)}=\Delta \gamma_{n+1}^{\alpha(k)}+\delta \gamma_{n+1}^{\alpha(k)}
\end{aligned}
$$

Set $k \leftarrow k+1$ and go to 4 . 